\title{
The Plasma Simulation Code: A modern particle-in-cell code with patch-based load-balancing
}

\author{
Kai Germaschewski ${ }^{\mathrm{a}, *}$, William Fox ${ }^{\mathrm{c}}$, Stephen Abbott ${ }^{\mathrm{d}}$, Narges Ahmadi ${ }^{\mathrm{a}}$, Kristofor \\ Maynard $^{\mathrm{a}}$, Liang Wang ${ }^{\mathrm{a}}$, Hartmut Ruhl ${ }^{\mathrm{b}}$, Amitava Bhattacharjee ${ }^{\mathrm{c}}$ \\ ${ }^{a}$ Space Science Center $\mathcal{E}$ Department of Physics, University of New Hampshire, Durham, NH \\ ${ }^{b}$ Faculty of Physics, Ludwig Maximilians University, München, Germany \\ ${ }^{c}$ Princeton Plasma Physics Laboratory, Princeton, NJ \\ ${ }^{d}$ Oak Ridge National Laboratory, Oak Ridge, TN
}

\begin{abstract}
This work describes the Plasma Simulation Code (PSC), an explicit, electromagnetic particle-in-cell code with support for different order particle shape functions. We review the basic components of the particle-in-cell method as well as the computational architecture of the PSc code that allows support for modular algorithms and data structure in the code. We then describe and analyze in detail a distinguishing feature of Psc: patch-based load balancing using space-filling curves which is shown to lead to major efficiency gains over unbalanced methods and a previously used simpler balancing method.
\end{abstract}

Keywords: particle-in-cell, kinetic, plasma, load balancing

\section{Introduction}

Rapidly advancing computer technology has enabled large first-principles plasma simulations in recent years. The kinetic description of plasmas, while computationally much more expensive, overcomes many limitations of fluid descriptions like magnetohydrodynamics (MHD) or extended MHD models. Fluid models describe plasma behavior at large scales very well, but approximations need to be made at small scales, which occur in magnetic reconnection and turbulence. For example, the one-fluid approximation breaks down at the ion skin depth scale $d_{i}$, electrons and ions decouple, and the magnetic field remains frozen to the electron flow. Reconnection requires breaking the frozen-in condition that occurs at electron scales, which can be represented in a fluid model in a generalized Ohm's Law that includes electron inertia and electron pressure tensor effects. Finding appropriate closures is still an area of active research, see e.g. [1]. The kinetic description of a plasma evolves the full 6-dimensional distribution function in time, either directly solving the Vlasov-Maxwell-Boltzmann equations (see e.g. [2]), or using the particle-in-cell method that this work focuses on. The particle-in-cell method is typically much less computationally intensive as it approximates the velocity part of the distribution function by quasi-particles which are evolved in 3-d configuration space, but also introduces complications like discrete particle noise, mapping between two spatial domains (a discrete mesh and continuous particle positions) and potential numerical instabilities). Kinetic models also allow

\footnotetext{
${ }^{*}$ Corresponding author

Email addresses: kai.germaschewski@unh.edu (Kai Germaschewski), wf ox@pppl.gov (William Fox), abbottsr@ornl .gov (Stephen Abbott), narges . ahmadi@unh.edu (Narges Ahmadi), k. maynard@unh.edu (Kristofor Maynard), liang. wang@unh.edu (Liang Wang), hartmut.ruhl@uni-muenchen.de (Hartmut Ruhl), amitava@princeton.edu (Amitava Bhattacharjee)
} 
investigation of problems entirely beyond the scope of fluid models, e.g. particle acceleration [3]. Particle-in-cell codes, while often run with modified physical parameters (e.g. reduced ion/electron mass ratio and speed of light), are now capable of simulating multi-scale problems spanning from electron through ion to global scales reaching 100's of $d_{i}$ in two and even three dimensions. While efforts are underway to overcome some of the algorithmic limitations of explicit particle-in-cell methods (see, e.g., [4, 5]), explicit particle-in-cell methods scale efficiently to the largest supercomputers available today and are commony used to address challenging science problems.

The Plasma Simulation Code (PSC) is an explicit, electromagnetic particle-in-cell code implementing similar methods as, e.g., VPIC [6], osIRIS[7] and vorPAL [8]. PSC is based on H. Ruhl's original version [9], but has been rewritten as modular code that supports flexible algorithms and data structures. Beyond its origin in the field of laser-plasma interaction, PSc has been used in studies of laser-produced plasma bubbles [10, 11, 12, 13], particle acceleration [14], and closure aspects in magnetic reconnection [15].

In this paper, we will review the main underlying particle-in-cell methods and the computational infrastructure of the PSC code. We will then introduce and analyze a distinguishing feature implemented in PSc: Patch-based dynamic load balancing addresses both performance and memory issues in simulations where many particles move between local domains.

\section{Particle-in-cell method}

\subsection{Kinetic description of plasmas}

The particle-in-cell method $[16,17,5]$ solves equations of motion for particles and Maxwell's equations to find forces between those particles, which is very similar to the first-principle description of a plasma as a system of charged particles. It is, however, better understood as a numerical method to solve the Vlasov-Maxwell system of equations that describes the time evolution of the particle distribution function $f_{s}(\mathbf{x}, \mathbf{p}, t)$ where $s$ indicates the species:

$$
\frac{\partial f_{s}}{\partial t}+\mathbf{v} \cdot \frac{\partial f_{s}}{\partial \mathbf{x}}+q_{s}(\mathbf{E}+\mathbf{v} \times \mathbf{B}) \cdot \frac{\partial f_{s}}{\partial \mathbf{p}}=0
$$

The electromagnetic fields $\mathbf{E}$ and $\mathbf{B}$ are self-consistently evolved using Maxwell's equations:

$$
\begin{aligned}
\nabla \cdot \mathbf{E} & =\frac{\rho}{\epsilon_{0}} \\
\nabla \cdot \mathbf{B} & =0 \\
\frac{\partial \mathbf{E}}{\partial t} & =c^{2} \nabla \times \mathbf{B}-\frac{\mathbf{j}}{\epsilon_{0}} \\
\frac{\partial \mathbf{B}}{\partial t} & =-\nabla \times \mathbf{E}
\end{aligned}
$$

where charge density $\rho$ and current density $\mathbf{j}$ are obtained from the particle distribution functions:

$$
\begin{aligned}
& \rho=\sum_{s} q_{s} \int f_{s}(\mathbf{x}, \mathbf{p}, t) d^{3} p \\
& \mathbf{j}=\sum_{s} q_{s} \int \mathbf{v} f_{s}(\mathbf{x}, \mathbf{p}, t) d^{3} p
\end{aligned}
$$

If the divergence equations (2), (3) in Maxwell's equations are satisfied at some initial time, it is easy to show from Ampére's Law (4) and Faraday's Law (5) that they will remain satisifed at all times provided that the charge continuity equation also holds:

$$
\partial_{t} \rho+\nabla \cdot \mathbf{j}=0
$$

This is important in the numerical implementation, where only Eqns. (4) and (5) are used to advance the electromagnetic fields in time. Even if Eqns. (2), (3) are discretely satisfied initially, large unphysical violations of Eqns. (2), (3) can accumulate, which is prevented here by using a compatible exact discrete implementation of Eqn. (8). 


\subsubsection{Particle-in-Cell method}

The particle-in-cell method approximates the distribution function $f_{s}$ by representing it using quasi-particles with finite extent in configuration space:

$$
f_{s}(\mathbf{x}, \mathbf{p}, t)=\sum_{i=1}^{N_{s}} N_{i}^{s} \phi\left(\mathbf{x}-\mathbf{x}_{i}^{s}(t)\right) \delta^{3}\left(\mathbf{p}-\mathbf{p}_{i}^{s}(t)\right)
$$

Using the $\delta$-function in velocity space ensures that the spatial extent of each quasi-particle remains constant in time.

The selection of the shape function $\phi$ determines properties of the numerical method. In general, the 3-d shape function is chosen to be the tensor product of 1-d shape functions in each coordinate direction; normalized, symmetric shape functions with compact support are used. Equations of motions for the quasi-particles can then be derived by taking moments of the Vlasov equation:

$$
\frac{d N_{i}^{s}}{d t}=0, \frac{d \mathbf{x}_{i}^{s}}{d t}=\mathbf{v}_{i}^{s}, \quad \frac{d \mathbf{p}_{i}^{s}}{d t}=q_{s}\left(\mathbf{E}_{i}+\mathbf{v}_{i}^{s} \times \mathbf{B}_{i}\right)
$$

The first equation expresses that the number of actual particles $N_{i}^{s}$ that each quasi-particle $i$ of species $s$ represents remains constant. The other two equations are the usual equations of motion for a point particle with the modification that the electromagnetic fields $\mathbf{E}_{i}, \mathbf{B}_{i}$ acting on the particle are given by

$$
\mathbf{E}_{i}=\int \mathbf{E} \phi\left(\mathbf{x}-\mathbf{x}_{i}^{s}\right) d^{3} x, \quad \mathbf{B}_{i}=\int \mathbf{B} \phi\left(\mathbf{x}-\mathbf{x}_{i}^{s}\right) d^{3} x
$$

which means that the electromagnetic fields are averaged over the extent of the particle.

Using finite-size quasi-particles is the main advantage of the particle-in-cell method. It is computationally cheaper, since it allows one to solve the field equations on a mesh, rather than directly calculating the interaction of each particle with all others. Particle-in-cell scales linearly in the number of particles $N$, as opposed to exact interaction approach that scales like $O\left(N^{2}\right)$ (though this can be improved to $O(N \log N)$ by fast multipole methods [18]). More importantly, even with today's very powerful computers it is in most cases not possible to simulate as many particles as comprise a plasma of interest, and it will remain unfeasible for the foreseeable future for, e.g., applications space physics and high-energy-density plasmas. In lowering the number of particles to a number that is possible to simulate one must be careful to not change the nature of the plasma. Plasmas are traditionally weakly coupled, ie. the interaction between particles is dominated by collective behavior rather than individual particle-particle forces. The particle-incell method reduces the occurence of strong particle-particle interactions because particles are now of finite extent, which means that their interaction potential weakens when two particles approach closer than their spatial size, while still representing the long-range interactions faithfully.

\subsection{FDTD method for solving Maxwell's equations}

The finite-difference time domain (FDTD) method has a long history of being used for computationally solving Maxwell's equations [19]. The FDTD method has the desirable feature of satisfying some conservation properties of the underlying continuum equations in the discrete. It employs the staggered Yee grid, as shown in Fig. 1, to represent magnetic fields on faces, electric fields and current densities on edges, and charge densities on corners of the computational mesh.

We define the following discrete curl operators:

$$
\begin{aligned}
\left(\nabla^{+} \times \mathbf{E}\right)_{x, i, j+1 / 2, k+1 / 2} & =\frac{E_{z, i, j+1, k+1 / 2}-E_{z, i, j, k+1 / 2}}{\Delta y}-\frac{E_{y, i, j+1 / 2, k+1}-E_{y, i, j+1 / 2, k}}{\Delta z} \\
\left(\nabla^{-} \times \mathbf{B}\right)_{x, i+1 / 2, j, k} & =\frac{B_{z, i+1 / 2, j+1 / 2, k}-B_{z, i+1 / 2, j-1 / 2, k}}{\Delta y}-\frac{B_{y, i+1 / 2, j, k+1 / 2}-B_{y, i+1 / 2, j, k-1 / 2}}{\Delta z}
\end{aligned}
$$

where the $y$ and $z$ components are obtained by cyclic permutation. 

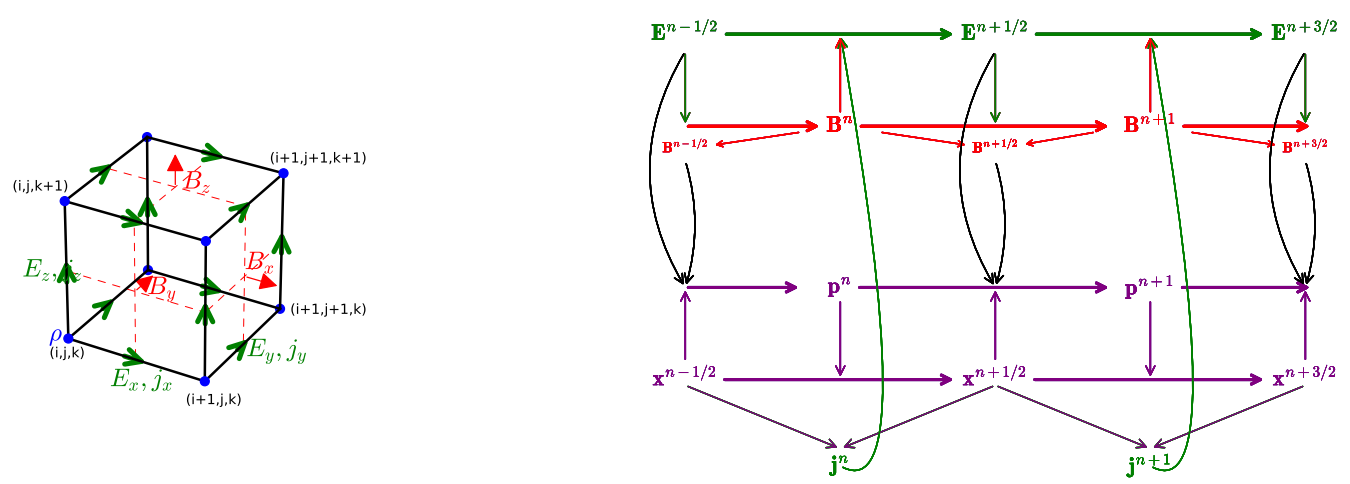

Figure 1. The staggered Yee grid unit cell. Depicted are the locations of magnetic fields on face centers (red), eletric fields and current density (green), and charge density (blue)
Figure 2. Leapfrog time integration in the PIC method. Blue quantities represent electromagnetic field quantities and their update scheme. Red quantities are quasi-particle positions and momenta, also staggered in time. Interaction occurs by using the EM fields to find the Lorentz force on particles (black) and by using particle motion to find current density that feeds back into Maxwell's equations (green).

We also define the following discrete divergence operators:

$$
\begin{aligned}
\left(\nabla^{+} \cdot \mathbf{B}\right)_{i+1 / 2, j+1 / 2, k+1 / 2}= & \frac{B_{x, i+1, j+1 / 2, k+1 / 2}-B_{x, i, j+1 / 2, k+1 / 2}}{\Delta x}+\frac{B_{y, i+1 / 2, j+1, k+1 / 2}-B_{y, i+1 / 2, j, k+1 / 2}}{\Delta y}+ \\
\left(\nabla^{-} \cdot \mathbf{E}\right)_{i, j, k}= & \frac{B_{z, i+1 / 2, j+1 / 2, k+1}-B_{z, i+1 / 2, j+1 / 2, k}}{\Delta z} \\
\Delta x &
\end{aligned}
$$

Maxwell's equations are discretized using these operators, and we employ a leapfrog scheme staggered in time (see also Fig. 2):

$$
\begin{aligned}
\frac{\mathbf{E}_{i j k}^{n+1 / 2}-\mathbf{E}_{i j k}^{n-1 / 2}}{\Delta t} & =c^{2} \nabla^{-} \times \mathbf{B}_{i j k}^{n}-\frac{\mathbf{j}_{i j k}^{n}}{\epsilon_{0}} \\
\frac{\mathbf{B}_{i j k}^{n+1}-\mathbf{B}_{i j k}^{n}}{\Delta t} & =-\nabla^{+} \times \mathbf{E}_{i j k}^{n+1 / 2}
\end{aligned}
$$

where

$$
\begin{aligned}
& \mathbf{E}_{i j k}=\left(E_{x, i+1 / 2, j, k}, E_{y, i, j+1 / 2, k}, E_{z, i, j, k+1 / 2}\right) \\
& \mathbf{B}_{i j k}=\left(B_{x, i, j+1 / 2, k+1 / 2}, B_{y, i+1 / 2, j, k+1 / 2}, B_{z, i+1 / 2, j+1 / 2, k}\right)
\end{aligned}
$$

It is easy to show that the discrete operators satisfy

$$
\nabla^{+} \cdot \nabla^{+} \times=0, \nabla^{-} \cdot \nabla^{-} \times=0
$$

and hence, as in the continuum, the discretized divergence equations remain satisfied to round-off error at all times,

$$
\left(\nabla^{-} \cdot \mathbf{E}\right)_{i j k}=\frac{\rho_{i j k}}{\epsilon_{0}}, \quad\left(\nabla^{+} \cdot \mathbf{B}\right)_{i+1 / 2, j+1 / 2, k+1 / 2}=0
$$


provided that the charge continuity equation is also discretely satisfied:

$$
\frac{\rho_{i j k}^{n+1 / 2}-\rho_{i j k}^{n-1 / 2}}{\Delta t}+\left(\nabla^{-} \cdot \mathbf{j}\right)_{i j k}=0
$$

The FDTD method also satisifies a discrete version of Poynting's Theorem; however, it involves $\mathbf{E}$ and $\mathbf{B}$ at staggered time levels. When used in the context of a PIC method energy is generally not exactly conserved because $\mathbf{j} \cdot \mathbf{E}$ is discretized differently in the Maxwell solver compared to the particle advance.

\subsection{Time integration of the quasi-particle equations of motion}

We use a standard leapfrog method to advance quasi-particles in time, see also Fig. 2.

$$
\begin{aligned}
\frac{\mathbf{x}_{i}^{n+1 / 2}-\mathbf{x}_{i}^{n-1 / 2}}{\Delta t} & =\mathbf{v}_{i}^{n} \\
\frac{\mathbf{p}_{i}^{n+1}-\mathbf{p}_{i}^{n}}{\Delta t} & =q_{s}\left(\mathbf{E}_{i}^{n+1 / 2}+\mathbf{v}_{i}^{n+1 / 2} \times \mathbf{B}_{i}^{n+1 / 2}\right)
\end{aligned}
$$

where $\mathbf{v}_{i}^{n}=\mathbf{p}_{i}^{n} /\left(m_{s} \gamma_{i}^{n}\right)$. We follow Boris [20] in choosing

$$
\mathbf{v}_{i}^{n+1 / 2}=\frac{\mathbf{p}_{i}^{n}+\mathbf{p}_{i}^{n+1}}{2 m_{s} \gamma_{i}^{n+1 / 2}}
$$

and splitting the momentum update into a half step acceleration by $\mathbf{E}$, a rotation by $\mathbf{B}$, and another half step acceleration by $\mathbf{E}$.

The shape functions used in the Psc code are standard B-splines $[5,16]$. The code currently supports both 1st and 2nd order interpolation by employing the flat-top $b_{0}(\xi) \mathrm{B}$-spline and the triangular-shaped $b_{1}(\xi) \mathrm{B}$-spline. $b_{0}(\xi)$ is defined as

$$
b_{0}(\xi)= \begin{cases}1 & \text { if }|\xi|<1 / 2 \\ 0 & \text { otherwise }\end{cases}
$$

Successive B-splines are defined recursively by folding the previous B-spline with $b_{0}$ :

$$
b_{n+1}=\int_{-\infty}^{\infty} b_{0}\left(\xi-\xi^{\prime}\right) b_{n}\left(\xi-\xi^{\prime}\right) d \xi^{\prime}
$$

In particular, the Psc code uses $b_{1}$ and $b_{2}$ :

$$
b_{1}(\xi)=\left\{\begin{array}{ll}
1+\xi & \text { if }-1 \leq \xi \leq 0 \\
1-\xi & \text { if } 0 \leq \xi \leq 1 \\
0 & \text { otherwise }
\end{array} \quad b_{2}(\xi)= \begin{cases}\frac{1}{2}\left(\frac{3}{2}+\xi\right)^{2} & \text { if }-\frac{3}{2} \leq \xi \leq-\frac{1}{2} \\
\frac{3}{4}-\xi^{2} & \text { if }-\frac{1}{2} \leq \xi \leq \frac{1}{2} \\
\frac{1}{2}\left(\frac{3}{2}-\xi\right)^{2} & \text { if } \frac{1}{2} \leq \xi \leq \frac{3}{2} \\
0 & \text { otherwise }\end{cases}\right.
$$

B-splines are commonly used in PIC codes because of their simplicity and compact support. Also, when assuming that the electromagnetic fields are piecewise constant about their staggered grid locations the integrals in Eq. 11 are conveniently evaluated and found to be B-splines themselves, of order one higher than the shape function itself. Hence the Psc code uses B-splines of order 1 and 2 to interpolate the electromagnetic fields to the quasi-particle position.

\subsection{Time integration}

The particle-in-cell method advances both electromagnetic fields and quasi-particles self-consistently. The time integration scheme used in PSc is sketched out in Fig. 2. The figure shows the FDTD scheme (blue), and particle integrator (red), and also their interactions: To update the momentum, the electric and magnetic fields are needed to find the force on a given quasi-particle (black arrows). $\mathbf{E}^{n+1 / 2}$ exists at the proper centered time to do so, while $\mathbf{B}^{n+1 / 2}$ 
is in principle found by averaging $\mathbf{B}^{n}$ and $\mathbf{B}^{n+1}$; however, in practice, we rather split the $\mathbf{B}^{n} \rightarrow \mathbf{B}^{n+1}$ update into two half steps.

Particle motion feeds back into Maxwell's equations by providing the source term $\mathbf{j}^{n}$. The current density is computed from the particles to exactly satisfy the discrete charge continuity equation, which requires knowing particle positions at the naturally existing $\mathbf{x}^{n-1 / 2}$ and $\mathbf{x}^{n+1 / 2}$, and is fed back into Maxwell's equations (green arrows).

PSC uses two methods to satisfy charge continuity: For 1st-order particles we use the scheme by VillasenorBuneman [21], while for 2nd-order particles we follow the method by Esirkepov [22]. PSC also implements some alternating-order interpolation schemes from [23] for improved energy conservation. For a discussion of conservation properties of particle-in-cell codes, see also [24].

We find that single precision simulations that run for a very large number of steps accumlate round-off errors that lead to growing deviations from the discrete Gauss's Law. Psc implements the iterative method by Marder [25] to dissipate violations of Gauss's Law.

\subsection{Collisions}

The PSC code implements a collision operator of the Takizuka-Abe (TA) family [26] to include binary Coulomb collisions; see e.g. [27] for an alternative approach. Collisional Psc has been used in a number of recent publications $[11,12,28]$ focused on kinetic interactions of plasmas, in particular in high-energy-density regimes driven by lasers. The dominant physics is kinetic, but collisions must be retained for complete fidelity to experiment.

In the TA-family of collision algorithms, particles in the same cell are randomly paired and "scattered" from one another, by exchanging momentum through a scattering angle. By appropriately choosing the pairing method and scattering angle, the algorithm can reproduce physical transport coefficients of a classical plasma. The collision algorithm exploits two features of screened Coulomb collisions in a plasma. First that typical interaction distances are of order the particle Debye length. Because the cell size in explicit particle-in-cell simulations is also about 1 Debye length (by numerical stability constraints), a convenient immediate choice is that particles are paired within cells for collisions. This conveniently makes the collision operator local, and can be implemented cell-by-cell, and readily vectorized. It does, however, require that the particles be pre-sorted by cell before the collision step.

Second, a particle undergoing Coulomb collisions executes a type of random-walk in velocity space though a very large number of small-angle collisions. This is made precise by the following argument, taking electrons as an example: typical particle interaction times within a collision are $\sim \lambda_{D} / v_{t e}=\omega_{p e}^{-1}$. Meanwhile 90-degree scattering times are much longer, $\tau_{e i}=\frac{12 \pi^{3 / 2}}{2^{1 / 2}} \frac{m_{e} T_{e}^{3 / 2} \epsilon_{0}^{2}}{n e^{4} \log \Lambda}$. This shows that there are $\omega_{p e} \tau_{e i} \sim n \lambda_{D}^{3} / \log \Lambda$ "micro-collisions" per 90-degree scattering time, which is a very large number since $n \lambda_{D}^{3}$ is large for a weakly-coupled, classical plasmas.

In the computational collision, the details of particle orbits and scattering by the intense near-fields of it scattering partner are replaced by a simple discrete momentum exchange. Notably, this can still correctly mimic the ultimate diffusive nature of classical transport (and achieve correct rates), provided $\tau_{e i} \gg \Delta t_{c}$, where $\Delta t_{c}$ is the time step between calls to the collision operator. This ensures that the computation collisions are also small angle, achieving the random-walk regime. In fact, $\tau_{e i} \gg \Delta t_{c}$ typically still allows significant latitude, for example we can choose an ordering $\tau_{e i} \gg \Delta t_{c} \gg \omega_{p e}^{-1}$, allowing the collision operator to be sub-cycled compared to the particle step $\Delta t$.

We now briefly review the details of the algorithm for completeness. The most recent version of the TA algorithm in PSC differs from the original PSC algorithm [9] in how the particles are paired for scattering. Instead of the intricate rules of Ref. [9] which separates particles by species, in each collision step each particle is paired and collided with simply one other particle. With this change, the scattering angles are calculated as follows to account for the new statistics:

For each collision pair, first a scattering parameter $v \Delta t_{c}$ is calculated as

$$
v \Delta t_{c}=\left(v_{0} \Delta t_{c}\right)\left(\frac{n_{t o t}}{n_{0}}\right)\left(\frac{q_{1}}{e}\right)^{2}\left(\frac{q_{2}}{e}\right)^{2}\left(\frac{m_{e}}{m_{12}}\right)^{2}\left(\frac{c}{v_{r}}\right)^{3 / 2},
$$

where $\left(v_{0} \Delta t_{c}\right)$ is the fundamental collision frequency for the simulation, multiplied by the time-increment between calls to the collision routine (with subcycling), $n_{t o t}$ is the summed density over all species in the cell, and $n_{0}$ is the characteristic (normalization) plasma density for the simulation. $q_{1}$ and $q_{2}$ are the charges of the two colliding 
particles, $m_{12}$ is the reduced mass, and $v_{r}$ is the relative speed of the two colliding particles. $v_{0}$ has the interpretation of the slowing down time of a fast electron with speed $c$, it and it can be calculated numerically as

$$
v_{0}=\frac{n_{0} e^{4} \ln \Lambda}{4 \pi \epsilon_{0}^{2} m_{e}^{2} c^{3}},
$$

however in practice in the simulation it is a free parameter. Practically, it is chosen according to the desired collisionality of the simulation via

$$
v_{0}=3 \sqrt{\frac{\pi}{2}}\left(\frac{T_{e 0}}{m_{e} c^{2}}\right)^{3 / 2} \frac{1}{Z_{e f f}} v_{e i},
$$

where $v_{e i}$ is the Braginskii electron-ion collision frequency. (Note this fixes a typographic error in equation (A3) of Ref. [11].) The factor $n_{t o t} / n_{0}$ accounts for the statistics of a single collision per particle per collision step. The speed dependence of this collision parameter is what successfully reproduces the characteristic energy dependence of Coulomb transport. Combined with the condition for diffusive transport, $v_{e i} \Delta t_{c} \ll 1$ this allows reproduction of classical transport rates.

To scatter a pair of particles, the collision operator first transforms to the center of mass frame of the particles and then rotates the relative velocity vector through angles $(\theta, \phi)$. The frame shift is calculated using relativistic kinematics to conserve the full relativistic momentum and energy of the colliding pair. The polar scattering angle is determined from the collision parameter,

$$
\theta=2 \operatorname{atan}\left(\sqrt{-0.5 v \Delta t_{c} \log (1-r)}\right)
$$

where $r$ is a uniform random variable on $[0,1)$. If $v \Delta t_{c}>1$, the scattering is a "large-angle" collision, discussed below, and the polar scattering angle is instead chosen for equal probability per unit solid angle. It can be verified that for $v \Delta t_{c}$ small, the distribution of scattering angles is a 2-d Gaussian with variance $v \Delta t_{c}$. Meanwhile the azimuthal angle is simply a uniform random variable on $[0,2 \pi)]$.

We now show convergence and document a useful practical in situ convergence diagnostic for running the algorithm in a complex evolving plasma simulation. Here, we test the convergence for the Spitzer resisitivity problem with respect to the parameter $v_{e i} \Delta t_{c}$. Here we use $\mathrm{Z}=1$, and use a single cell simulation, with 100k computational particles (50k electrons, 50k ions), similar to the test demonstrated in Ref [11]. Particles are initialized with a Maxwellian distribution with temperature $T_{0}$, an electric field $E$ is applied, and we then execute the PIC algorithm and collision operator within the cell. After a few collision times, the distributions achieve a steady state, with total current approximately equal to the Spitzer current $J_{s p}=1.98 n e E / v_{e i}$, where we track the small change in $v_{e i}$ due to $J \cdot E$ heating during the simulation. During the run, we record additional statistical output, namely, median $\left(v \Delta t_{c}\right)$, the median scattering parameter over all collision pairs, and fraction $(L A)$, the fraction of "large-angle" collisions, for which $v \Delta t_{c}>1$. (The latter indicates breakdown of the diffusive condition.) We also record the resulting error $J / J_{s p}-1$. The results are summarized in Table 1. The error is found to increase with increasing $v_{e i} \Delta t_{c}$, however, we note the algorithm never fails catastrophically: even at $v_{e i} \Delta t_{c}=0.2$, where close to $40 \%$ of all collisions are large-angle collisions, the error in the details of the Spitzer current is only about $20 \%$. The results show that median $\left(v \Delta t_{c}\right)$ and fraction $(L A)$ are useful in in situ diagnostics to monitor fidelity of the collision algorithm in a complex evolving plasma simulation, where densities and temperatures may differ significantly from nominal values. The rule of thumb is that the error in transport coefficients is comparable to median $\left(v \Delta t_{c}\right)$. This allows selection of appropriate collision operator subcycling $\left(\Delta t_{c} / \Delta t\right)$ in situations where $v_{e i} \ll \omega_{p e}$ to speed up the computational algorithm while maintaining physics fidelity.

The Psc code supports a number of additional features, including periodic, reflecting conducting wall, and open boundary conditions (similar to [29]), moving window and boost frame, which are not addressed in detail here.

\section{Overview of the PSC code}

The Plasma Simulation Code (PSC) presented in this paper is based on the original Fortran code by H. Ruhl [9], but it has been largely rewritten. The original Fortran computational kernels (particle advance, FDTD Maxwell solver) are still available as modules, but the code's overall framework is now written in the C programming language. The open-source version of the code, described here, is maintained by the group at the University of New Hampshire; 
Table 1. Collision diagnostics

\begin{tabular}{cccc}
\hline \hline$v_{e i} \Delta t_{c}$ & median $\left(v \Delta t_{c}\right)$ & fraction $(L A)$ & $\left(J / J_{s p}-1\right)(\%)$ \\
\hline 0.02 & 0.028 & 0.06 & $+3 \%$ \\
0.05 & 0.07 & 0.15 & $+7 \%$ \\
0.1 & 0.14 & 0.26 & $+15 \%$ \\
0.2 & 0.28 & 0.45 & $+20 \%$ \\
\hline \hline
\end{tabular}

development has been performed in collaboration with H. Ruhl's group at Ludwig Maximilian University of Munich. That group has added proprietary features to the code (e.g., quantum electrodynamics processes at high field strengths), but the basis is shared with the open-source version.

The structure of the code is based on LIBMRC, a parallel object model and library that forms the basis of a number of simulation codes maintained by the author, including the Magnetic Reconnection Code (MRCv3) [30, 31, 32] and J. Raeder's global magnetosphere code OPENGGCM [33, 34]. We consider LIBMRC to be a library rather than a framework, because it consolidates commonly used computational techniques in order to avoid reimplementing and maintaining common tasks like domain decomposition and I/O in individual codes. It is designed so that only selected parts of it can be used (e.g., filling of ghost points) in an otherwise legacy code without requiring large changes to the structure of the code overall. LIBMRc is licensed under the GNU Lesser General Public License (LGPLv3), while Pscis available under the GNU General Public License (GPLv3). Stable and development branches are available at [35].

LIBMRC is written in $\mathrm{C}$, but supports Fortran-order multidimensional arrays to enable easy interfacing with existing Fortran code. Its basis is an object model that is quite similar to the one used in PETSc [36, 37, 38] - and LIBMRC can optionally interface with PETSc to provide linear and nonlinear solvers, etc., though this feature is not used in the PSc code. Objects can be instantiated in parallel, i.e. they have an MPI communicator associated with them. Objects instantiate a given class, which essentially defines an interface. In the PIC context, for example, this may be a field pusher which provides two methods to update electric and magnetic fields, respectively. There may be more than one implementation for a given class, which we call "subclasses" or "types". In the example of the field pusher, this may be a single precision or double precision implementation of the FDTD method, or it could potentially encompass methods other than FDTD. In the case of the particle pusher, there are types for first and second order particle shapes, or a particle pusher that runs on GPUs. Like PETSc objects, the type of a given object can be set at run time potentially from a command line option, which allows the user to easily switch out modules in a given run.

The LIBMRC library provides a number of classes for common computational tasks, e.g. a parallel multi-dimensional field type which is distributed amongst MPI processes and associated coordinates. It handles parallel I/O; currently implemented options include the simple "one file per MPI process" approach as well as parallel XDMF/HDF5 [39] output using a subset of I/O writer nodes. As we will explain in more detail in the load balancing section, LIBMRC fields can be decomposed into many "patches", where a given MPI process may handle more than one patch - the very same interface is used to support block-structured adaptive mesh refinement, where again a given process handles multiple patches which are possibly at different levels of resolution. LIBMRC objects maintain explicit information about their own state, e.g. an object knows about member variables that are parameters, so these can be automatically parsed from the command line. This also simplifies checkpoint / restart: Every object knows how to write itself to disk, and how to restore itself, which means that writing a checkpoint just consists of walking down the hierarchy of objects asking each object to checkpoint itself.

The PSC code uses LIBMRC objects extensively: There are objects for all computational kernels (particles, fields), particle boundary exchange / filling ghost points, outputting fields and particles, etc. All these objects are contained within one psc object that represents the overall simulation. To implement a particular case one "derives" from the psc object, i.e. one implements a particular subclass. In this subclass one can overwrite various methods as needed - the create () methods to set defaults for domain size, resolution, normalization, particles species, etc., the init_fields() method to set initial conditions, and similarly an init_npt() method to set the initial condition for particles.

The aforementioned objects are primarily used to select particular algorithms, i.e. a second or first order particle 
pusher has little or no state associated with it but rather just implements a different computational algorithm. The simulation state is maintained in two additional objects: psc_fields and psc_particles, which are the large distributed arrays that represent the field and particle state. Those objects themselves may actually be implemented as rather different data structures: The particle data may be a simple array of struct in double precision living in CPU memory, but it can also be a more complicated struct of arrays of small vectors in GPU memory in single precision, just by selecting its subclass to be either "double" or "cuda".

The flexibility of supporting multiple data layouts is crucial to supporting both CPUs and GPUs in one code, but it also presents a significant challenge in implementing the algorithms that actually work on that data. For example, for obvious reasons a CPU particle pusher will not work when the particle data passed to it actually lives in GPU memory and/or is in the wrong layout. In traditional object oriented programming the solution to this is to not access data directly, but to virtualize it through methods of the particle object. However, for a high-performance code it is not acceptable to abstract all accesses through virtual (indirect) method calls because of the performance penalties incurred. Different approaches exist to overcome this hurdle.

One solution is to only support a matching set of modules - double precision CPU particle pusher with double precision CPU particles and double precision CPU fields. However, this means one essentially has to rewrite the entire code to support, e.g., a GPU implementation, which is a large effort and can easily lead to maintenance problems as CPU and GPU capabilities of the code can diverge.

Another option is to implement abstraction in a way that can be resolved at compile time, for example using some flavor of automatic code generation or template meta-programming in $\mathrm{C}++$. This approach has been successfully used by a number of particle-in-cell codes [40, 41, 42, 43]. One drawback is that it involves highly sophisticated programming techniques that many science users are not familiar with, which makes it difficult for them to customize and debug a code.

PSC chooses a different way to handle this problem: A particle pusher expecting double precision particles on the CPU needs to wrap its computations inside a pair of particles_get_as ("double") and particles_put_as () calls. The particle data structure returned from the get_as () call is guaranteed to be of the requested type, so the actual computation can be performed by directly accessing the known data structures without any performance penalties. Behind the scenes, the get_as () and put_as () calls perform conversion of the data structures if needed - if the particle data was actually stored as single precision it would be converted to double precision first, and the result will later (in put_as ()) be converted back. If the particle data was already of the type requested, get_as () and put_as () perform no actual work.

The main advantage to this approach is that it is now possible to implement new computational kernels one at a time, while keeping the overall code functional. There is of course a performance penalty for the data layout conversion, and it is typically severe enough that, for production runs, one wants to select a matching set of modules, e.g. particle and field data, particle pusher, and field pusher all of the "cuda" type for running on the GPU. Still, the main computational kernels are only a small fraction of the code overall, and other functionality like I/O and analysis often occur rarely enough that for those routines the conversion penalty is small, so it is not necessary to rewrite them for the new data types.

It should be noted that while a particle pusher on the CPU looks quite different from that on the GPU (so there is only limited room to share code), a second order pusher on the CPU working on single precision data is very similar to the same in double precision, so in this case we use a shared source file that gets compiled into a single and a double version by using the C preprocessor. While we end up with two distinct subclasses ("2nd_single" and "2nd_double"), we avoid unnecessary code duplication.

As mentioned before, output is typically written as XDMF/HDF5, which allows directly visualizing the data with Paraview, though we typically use custom scripts in Python or Matlab to downscale the resolution and perform specific analyses.

\section{Parallelization and load balancing}

\subsection{Parallelizing particle-in-cell simulations}

Due to their dual nature, particle-in-cell simulations are inherently more difficult to parallelize than either purely mesh-based or purely particle-based algorithms. For both mesh-based and particle-based methods a data parallel 


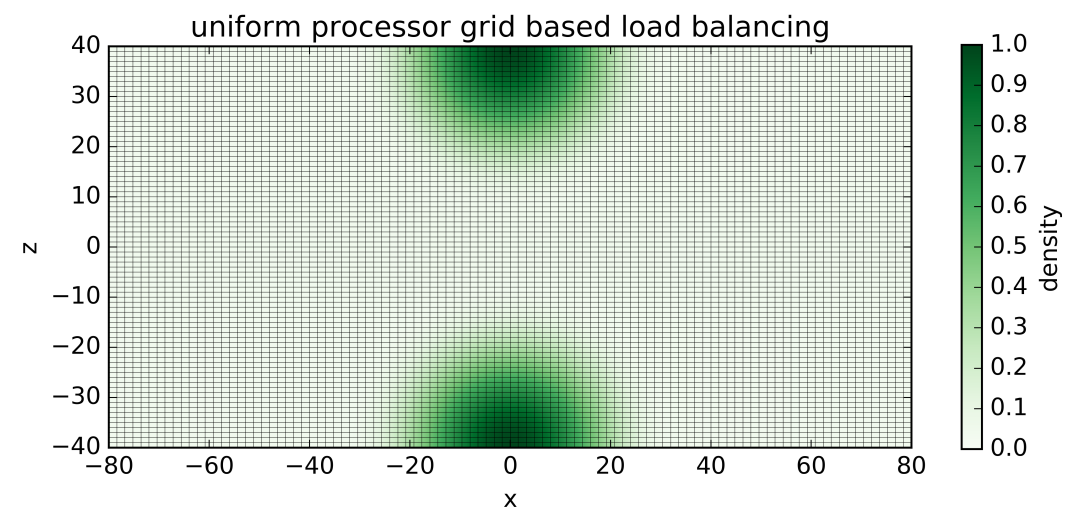

Figure 3. Initial condition for a laser-generated plasma bubble simulation. Shown are the plasma density (green) and the uniform domain decomposition of the computational domain into $100 \times 80$ patches.

approach is fairly straightforward, but the two-way interactions between fields and particles in the PIC method requires an approach that takes these interactions into account. In the following, we consider parallelization on a distributed memory machine using the message passing paradigm. Virtually all large supercomputers follow this paradigm as the coarse level of parallelism. Lower levels, like shared memory parallelization on a node or small vector instructions on a core, need to also be used but will be discussed later.

In a mesh-based simulation the typical approach to parallelization is domain decomposition: the spatial domain is subdivided into smaller subdomains, each subdomain is assigned to a different processing unit and processed separately. This works well if the computation is local in space, e.g. a stencil computation with a small stencil, as is the case for the FDTD scheme employed in PSc. Near the subdomain boundaries some data points from neighboring subdomains are required to update the local domain; these need to be communicated by message passing and are typically handled by a layer of ghost cells (also called halo regions). Domain decomposition for structured grids is a well established method and scales well as long as the boundary-related work remains small compared to the bulk computation, i.e. as long as the surface-to-volume ratio remains small.

In purely particle-based simulations, distributing particles in a data-parallel way is fundamentally quite simple, by just assigning subsets of particles to processors; however, interactions between particles will need to be taken into account and depending on their nature can make it rather challenging to find a parallel decomposition that still allows for efficient computation of those interactions, e.g., in the case of the fast multipole method [44].

For particle-in-cell simulations, interactions happen between particles and fields but not between particles and particles directly. An exception is the implementation of a collision operator, which approximates interactions of close-by particles by randomly picking representative particle pairs that are in close spatial vicinity.

Performance of particle-in-cell simulations is normally dominated by particle-related computational kernels rather than field computations, simply due to the fact that there are typically 100 or more particles per grid cell. Although, this assumption is not always true, in particular in a local sense. Simulations of laser-plasma interaction may have a significant fraction of the simulation that represent light waves in vacuum, and simulations of magnetic reconnection like-wise may have spatial regions at low density that are represented by just a few particles per cell.

Given these constraints, two approaches have been popular to parallelize particle-in-cell simulations on distributed memory machines:

(1) Distribute particles equally between processing units and redundantly keep copies of the fields on all processing units.

(2) Use a spatial decomposition of the domain and distribute particles according to which subdomain they are located in, so that particles and fields are maintained together on the same processing unit.

The main advantage to method (1) is its simplicity. Particles can be pushed independently of where they are located 
in the domain since all fields are available. As they move, current or charge density is deposited into the corresponding global fields. There are no load balancing issues - particles are distributed to processes equally in the beginning, and since they never move between processes this balance is maintained. The main drawback in this scheme is that the source terms for Maxwell's equations need to include contributions of all particles, so a global reduction for each global grid point is required at each time step. Maxwell's equations can be solved on one processor and the resulting fields broadcast to all processes, or the aggregated source field(s) can be broadcast and the computation performed redundantly on all processing units. The large, global reductions severely limit the parallel scalability and limit the applicability of the scheme to at most 100 s of cores.

Method (2) overcomes the scalability limitations of the previous scheme and is the approach used in state-of-theart codes like VPIC [6], osIRIs [7], PSc, and many others. Its implementation is more involved - particles will leave the local subdomain and need to be communicated to their new home. The field integration is performed locally on the subdomain and is therefore scalable. Both field integration and particles moving near boundaries require appropriate layers of ghost cells, and near subdomain boundaries proper care needs to be taken to correctly find contributions to the current density from particles in a neighboring subdomain, whose shape functions extend into the local domain.

Relativistic, explicit, electromagnetic particle-in-cell codes that follow the latter parallelization approach generally show excellent parallel scalability. Recently, osIRIs [7] has shown to scale to the full machine on NSF's Bluewaters Cray supercomputer, and PICONGPU has shown to scale to the full size of DOE's Titan machine while efficiently using the GPUs [41]. Fundamentally, this is easily explained by the underlying physics: Both particles and waves can propagate at most at the speed of light - since the time step is constrained by the CFL condition, information is guaranteed to propagate at most one grid cell per time step. Therefore interactions in the interior of the local subdomain happen entirely locally, and at subdomain boundaries only nearest-neighbor communication is required.

Other than the complexity of effectively implementing this parallelization method, there is one important drawback: It is hard to provide and maintain proper load balance in this method. As long as the plasma density throughout the simulation remains approximately uniform the number of particles assigned to each subdomain will be roughly constant and the simulations will perform well. In many cases, however, the initial density distribution may not be uniform. Even if it is initially, in many application areas like magnetic reconnection or laser-plasma interaction it will not remain that way as the simulation proceeds. Fig. 3 shows an example that we will analyze in more detail later: a simulation of expanding laser-generated plasma bubbles. Using a uniform domain decomposition (as indicated by the black mesh) some subdomains covering the bubbles have substantially higher density than the surrounding plasma, hence contain many more particles.

At best the ensuing load imbalance will just cause a performance slow-down. At worst it can lead to the code crashing with out-of-memory conditions as some local subdomains may accumulate more particles than there is memory available to hold them - this problem occured in some of our bubble reconnection simulations using the original version of PSC, and is exacerbated when using GPUs because they typically provide less main memory than a conventional multi-core compute node.

A number of approaches to load balancing PIC simulations have been used in the past. The easiest approach is to simply shift partition boundaries, although in general this does not offer enough degrees of freedom to always achieve good balance [9]. A more flexible approach is to partition the domain amongst one coordinate direction first to obtain columns with approximately balanced load. The next step then partitions columns in the next coordinate direction in order to obtain subdomains with approximately equal load. This scheme can work well, but it leads to subdomains with varying sizes and complicated communication patterns, as subdomains may now have many neighbors [45, 46]. A number of balancing schemes have been proposed in [47]; however these have not been implemented in actual PIC simulation codes. Here, we propose a new scheme, patch-based load balancing, which is similar to the method often used to parallelize adaptive mesh refinement methods, as it is for example in the WARP [48] particle-in-cell code.

In the following, we will analyze the factors that determine the performance of a particle-in-cell simulation, and then compare three different options for load balancing based on case studies of actual production simulations.

\subsection{Performance factors for a particle-in-cell simulation}

Factors that determine the performance of a typical particle-in-cell simulation are best explained using performance data from a sample run, as shown in Fig. 4.

The performance measurements are plotted as a function of timestep. The thick jagged green, red, and blue lines are all measurements of the execution time for the particle push (including current deposition). The green curve 


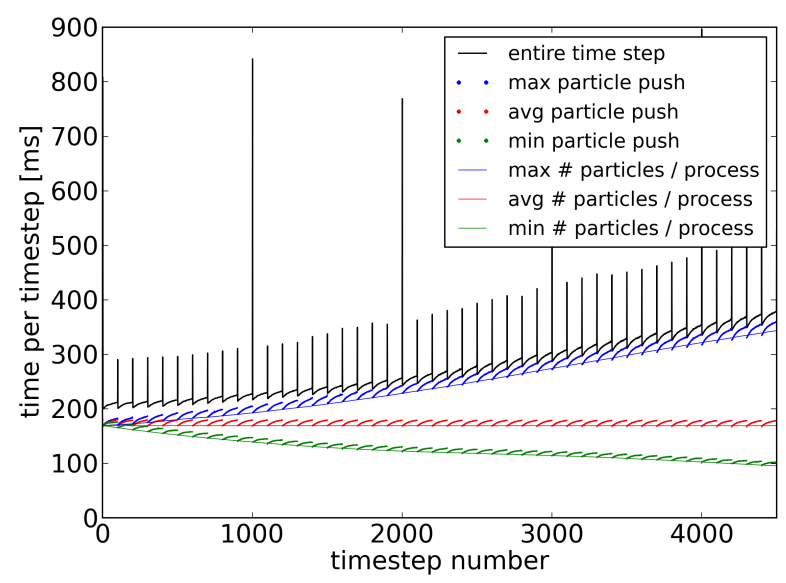

Figure 4. Computational performance over the course of a sample PIC simulation. Shown are particle push computation time at each step, min/average/max over all processes (thick green, red, blue curves), corresponding rescaled number of particles per process (thin green, red, blue curves), and total time per timestep (black).

gives the measurement from the fastest MPI process, the blue curve shows the slowest MPI process, and the red line indicates the average time over all processes. All measurements coincide in the beginning of the simulation. As the simulation runs there is an increasing spread between slowest and fastest processes, while the average performance remains approximately constant. The reason for this becomes clear when considering the number of particles on each process, which are plotted as the thin green, red, and blue lines. These data were rescaled to match the initial partitle push time. Initially, the number of particles handled by each process are equal in this simulation, but they then become unbalanced with some processes handling fewer than average, others handling more than average. The average particle number itself of course remains constant. The cause for the divergent particle push performance is clear: Particles move between MPI process boundaries, leaving some processes with more computational work in the particle pusher, and others with less.

In black we plot the total time per timestep - this number does not vary much between different processes, since processes that finish their work faster still have to wait for others to finish before communication can be completed. As expected, the total time per timestep tracks the slowest process. While unfortunate, it is the weakest link that determines overall performance, and that is why an unbalanced simulation can slow down a run substantially.

More can be learned from the data: Particle push time creeps up slightly until it suddenly falls back down to the expected level every 100 steps. That is because we sort particles by cell every 100 steps in this run - processing particles in sorted order is more cache-friendly, since $\mathbf{E}$ and $\mathbf{B}$ fields used to find the Lorentz force will be reused for many particles in the same cell before the pusher moves on to the next cell. The cost of sorting can also be seen in the total time per timestep as the small spikes in total time (black) every 100 steps. Additionally, we see larger spikes in total time every 1000 steps. These are caused by performing I/O.

\subsection{Dynamic load balancing using space filling curves}

In this work, we present a new patch-based approach to load balancing particle-in-cell simulations and investigate the performance costs and benefits.

The idea is easily stated: Given a number of processing elements, $N_{\text {proc }}$, decompose the domain into many more patches $N \gg N_{\text {proc }}$ than there are processing elements, and hence have each processing element handle a number of patches, typically $10-100$. By dynamically shifting the assigment of patches to processing elements we can ensure that each processing element is assigned a nearly equal load.

We will start by demonstrating the idea in a number of idealized cases. Later, we will analyze how it performs in real-world productions. 
(a)

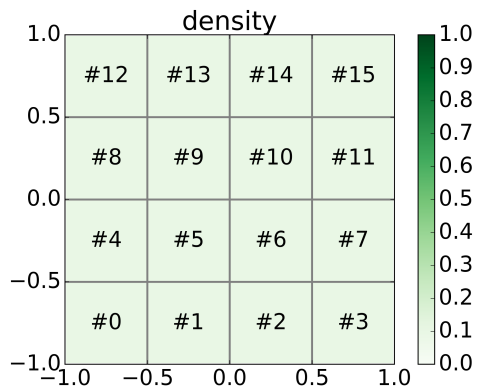

(b)

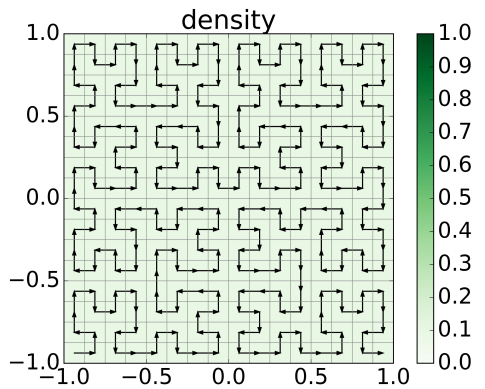

(c)

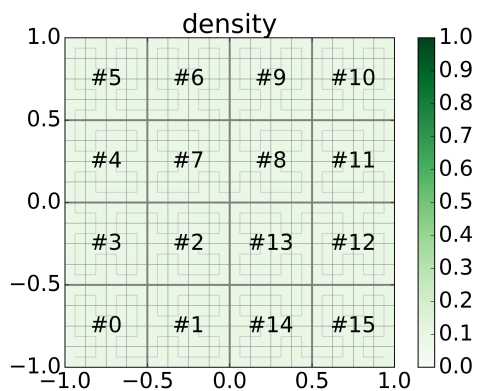

Figure 5. Basics of patch-based load balancing. The example simulation has uniform density and is parallelized to use 16 MPI processes. (a) Traditional domain decomposition into $4 \times 4$ subdomains. (b) Patch-based decomposition: The domain is now decomposed into $16 \times 16$ patches, and a Hilbert-Peano curve is used to assign patches to MPI processes. (c) Resulting assignment of the domain to MPI processes.

\subsubsection{Example: Uniform density}

The basic idea for load balancing by using many patches per processor is demonstrated in Fig. 5. We start out with a case of uniform density. We use 16 MPI processes to run the simulation, and use standard domain decomposition to divide the domain into $4 \times 4$ subdomains, one on each rank. This case is of course trivially load balanced already, since every subdomain is the same size and contains the same number of particles, see Fig. 5(a).

Still, to demonstrate our approach, we divide the domain into $16 \times 16$ subdomains instead, as shown in Fig. 5(b). Since there are now many more patches (256) than processes (16), each process needs to handle multiple patches, and it is necessary to define a policy that assigns patches to processes. Fig. 5(b) also shows the Hilbert-Peano spacefilling curve $[49,50]$. Following along the 1-d curve, each patch is visited exactly once. The 256-patch long curve is then partitioned into as many segments as we have processes, in this case we obtain 16 segments of 16 patches each. The segments of patches are then successively assigned to each MPI process. In the end (see Fig. 5(c)), we end up with essentially the same spatial decomposition as the standard partitioning using $4 \times 4$ subdomains, but we gained additional flexibility to react to changing loads by moving patches from processes with higher load to neighboring processes with lower load.

Choosing the Hilbert-Peano curve is just one option; one could, e.g., choose a simple row-major enumeration of the patches instead. As we will show later, the property that points which are close in 2-d (or 3-d) space are (on average) also close on the Hilbert-Peano curve does generally provide decompositions where the subdomain handled by a single MPI process is clustered in space, so that most communication between patches is actually local and does not require MPI communication, so that overall inter-process communication still benefits from scaling as the surface-to-volume ratio. This is the reason why space-filling curves have commonly been used to load balance blockstructured adaptive mesh refinement codes [51, 52, 53], and why, as we will show, it also works well for load balancing particle-in-cell simulations.

Up to this point we have only considered a case with uniform density (which is trivially load balanced) and so our load-balancing approach does not offer any major benefit here. We have shown, that it creates a good decomposition; essentially the same that one would have chosen in a one subdomain per process approach. There are actually some potential benefits, though, that are worth mentioning: The requirement to have a specific number of processes, e.g. square numbers like $4 \times 4$ can be abandoned - the same decomposition into 64 patches can be run on $64,16,15$, or 2 processes. We envision this to be a useful feature when dealing with node failures, which are expected to become a more common problem as simulations use an increasing numbers of cores as machine performance moves towards the exascale. If one node, say 32 cores, dies in a 250,000 core run, the code would be able to continue the simulation on just 249,968 cores by redistributing patches among the remaining cores - though obviously the data on those patches need to be recovered first, which requires some kind of frequent (possibly in-memory) checkpointing.

While having many small patches on a process means increased synchronization work at patch boundaries (in particular handling ghost points and exchanging particles), most of this work is wholly within the local subdomain and so can be handled directly or via shared memory rather than by more expensive MPI communication. As we will show later, dividing the work into smaller patches can even have a positive impact on computational performance due to enhanced data locality which makes better use of processor caches. 
(a)

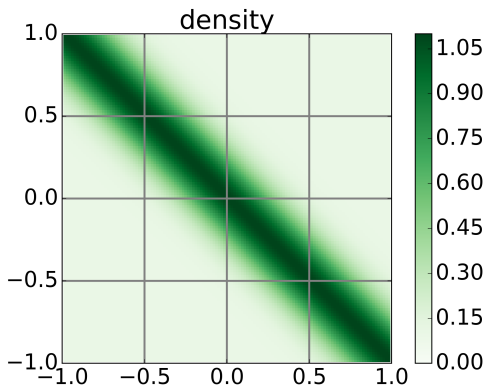

(d)

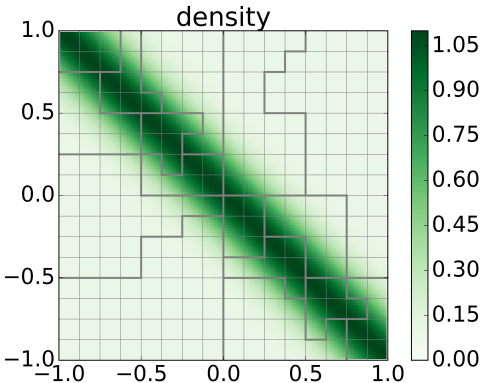

(b) 1.0

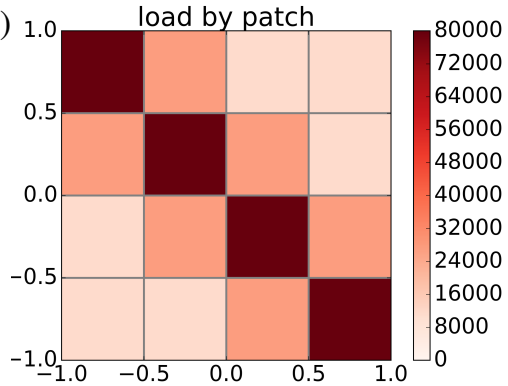

(e)

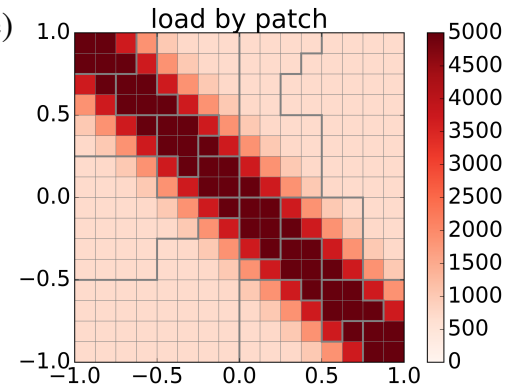

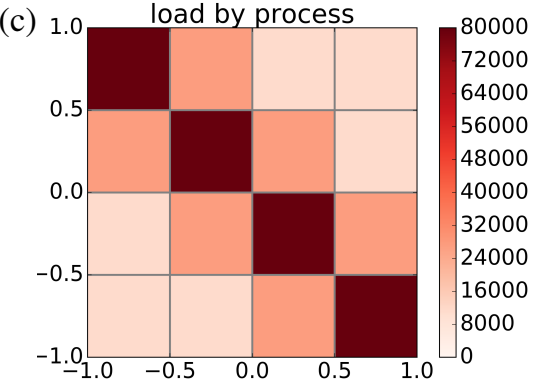

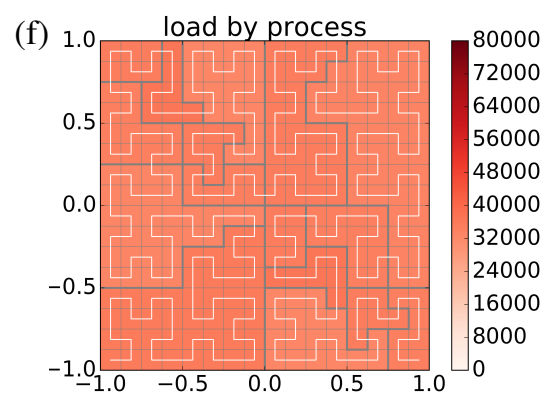

Figure 6. Load balancing increased density across the diagonal. We compare traditional domain decomposition (top row) to patch-based load balancing (bottom row). Thick black lines demarcate the subdomains assigned to each MPI process, thin grey lines show the underlying patches. (a), (d) plasma density. (b), (e) computational load for each decomposition unit. (c), (f) aggregate computational load for each MPI process. Computational load in this simple example is defined as number of particles.

\subsubsection{Example: Enhanced density across the diagonal}

The next example demonstrates the load balancing algorithm in action. We chose a uniform low background density of $n_{b}=0.1$ and enhance it by up to $n=1.1$ along the diagonal of the domain, as shown in Fig. 6 . This density distribution is chosen to be one where the Psc's former approach to load balancing is not effective at all: After dividing the domain into $4 \times 4$ subdomains it is not possible to shift the subdomain boundaries in a way that reduces load imbalance; this simulation will always be unbalanced by a factor of more than $7 \times$ as shown in Fig. 6(b), (c), where the load is simply defined as the number of particles per patch or subdomain, respectively. Subdomains near the high-density diagonal have an estimated load of 81200, while away from it the load is as low as 11300 .

Patch-based load balancing, however, works quite well. The resulting decomposition is shown in Fig. 6 (d)-(f) by the thicker black lines. It can be seen that some subdomains contain only a few patches, including some with a large load (high density, i.e. many particles), while other subdomains contain more patches but mostly at a low load.

Fig. 6 (b) shows the load for each patch, which is calculated for each patch as number of particles plus number of cells in that patch - clearly this mirrors the particle density. Fig. 6 (c) plots the aggregate load per process, calculated as the sum of the individual loads for each patch in that process's subdomain. It is clear that the load is not perfectly balanced, but it is contained within $\pm 7.5 \%$ of the average load of 29300 . This is certainly a vast improvement over an imbalance by a factor of more than $7 \times$ in the original code.

\subsubsection{Load balancing algorithm}

The goal of the actual balancing algorithm is quite straightforward: Divide the 1-d space filling curve that enumerates all patches into $N_{\text {proc }}$ segments, where $N_{\text {proc }}$ is the number of processes such that all processes have approximately equal load. In order to accomodate inhomogeneous machines, we add a "capability" specification. For example, we may want to run 16 MPI processes on a Cray XK7 node. 15 of those processes run on one core each, while the last one is used to drive the GPU on the node. In this case, we would assign a capability of 1 to the first 15 processes, and a capability of 60 to the last process, since the Titan's GPU (Nvidia Tesla K20X) performance is roughly $60 \times$ faster than a single CPU core for our code and we want it to get correspondingly more work.

The balancing algorithm hence divides the space-filling curve into segments that approximately match the capa- 


\begin{tabular}{|c|c|}
\hline $\begin{array}{l}\text { push_field_E_half(); } \\
\text { fill_ghosts_E(); } \\
\text { push_field_B_half(); } \\
\text { fill_ghosts_B(); } \\
\text { foreach(particle prt) \{ } \\
\quad \text { push_particle_x_half(prt); } \\
\text { push_particle_p(prt); } \\
\text { push_particle_x_half(prt); } \\
\text { push_particle_x_half_temp(prt); } \\
\text { deposit_j(); } \\
\text { \} } \\
\text { exchange_particles }() ; \\
\text { push_field_B_half(); } \\
\text { fill_ghosts_B(); } \\
\text { add_and_fill_ghosts_j(); } \\
\text { push_field_E_half }() ; \\
\text { fill_ghosts_E(); }\end{array}$ & $\begin{array}{l}/ / \mathbf{E}^{n} \rightarrow \mathbf{E}^{n+1 / 2} \\
/ / \text { communicate } \\
/ / \mathbf{B}^{n} \rightarrow \mathbf{B}^{n+1 / 2} \\
/ / \text { communicate } \\
/ / \mathbf{x}^{n} \rightarrow \mathbf{x}^{n+1 / 2}, \text { save for current } \\
/ / \mathbf{p}^{n} \rightarrow \mathbf{p}^{n+1} \\
/ / \mathbf{x}^{n+1 / 2} \rightarrow \mathbf{x}^{n+1} \\
/ / \mathbf{x}^{n+1} \rightarrow \mathbf{x}^{n+3 / 2}, \text { use for current, then disregard this update } \\
/ / \text { charge conservative current deposition using } \mathbf{x}^{n+1 / 2} \text { and } \mathbf{x}^{n+3 / 2} \\
/ / \text { communicate } \\
/ / \mathbf{B}^{n+1 / 2} \rightarrow \mathbf{B}^{n+1} \\
/ / \text { communicate } \\
/ / \text { communicate } \\
/ / \mathbf{E}^{n+1 / 2} \rightarrow \mathbf{E}^{n+1} \\
/ / \text { communicate }\end{array}$ \\
\hline
\end{tabular}

Figure 7. Original implementation of the timestep in PSc. All quantities start at time $t^{n}$ and are advanced to time $t^{n+1}$. Communication is performed at five different synchronization points.

bility for each rank - in the simple case of a homogeneous machine all capabities are equal and the algorithm reduces to distributing the load equally. The algorithm is described in more detail in Appendix A.

\subsubsection{Synchronization points}

As previously laid out, a time step in the PSc consists of a number of substeps that advance electric and magnetic fields and update particle positions and moments. Substeps depend on results from previous substeps, often not only within the local domain but (near the boundaries) also on results from remote processes. Hence communication is required and introduces synchronization points between processes, which interferes with load balancing the entire step.

While the PIC algorithm we use is naturally staggered in time for both particles and fields, the implementation in the original PSc broke up all but one step into two half steps so that the timestep would start with all quantities known at time $t^{n}$, and advance them all to time $t^{n+1}$, as shown in Fig. 7.

Communication occurs at five different points during the timestep as indicated, separated by computational kernels on either field or particles. Communication is implemented using non-blocking MPI send and receive calls; the time while messages are in flight is used to exchange field boundary data and particles between local patches that do not require inter-process communication. This was enough to hide the latency in our tests, so we do not implement overlap with computation on patches once they have all their boundary data set, as in [40, 41], though it could be added. However, these communications still introduce synchronization points. A given process will not be able to, e.g., update the fields until current density data has been received from neighboring processes. Neighboring processes won't be able to send these data until they finished pushing their local particles. The consequence is that it is not enough to just balance the total computational work, which includes both particle and field work. Rather, it is necessary to balance both particle work and field work individually between all processors. However, this is in general not possible.

In practice, experiments showed that, for typical cases, using our approach to load balancing still worked quite well because performance is dominated by particle work, with field work being comparatively fast, so that imbalance in the field work does not cause a great loss in performance. We set up the load balancing to equally distribute the number of particles that each process handles, up to the patch granularity. In a typical case we observed a slow-down of particle-dependent kernels by about $15 \%$ over the course of the run, which is consistent with the $15 \%$ deviation in particle number balance the algorithm achieved. The overall performance, however, would slow down by $30 \%$. Using the previous approach to load balancing by shifting process boundaries we observed a $200 \%$ slow-down in the same 


\begin{tabular}{|ll|}
\hline $\begin{array}{l}\text { push_field_B_half(); } \\
\text { foreach(particle prt) }\{\end{array}$ & $/ / \mathbf{B}^{n} \rightarrow \mathbf{B}^{n+1 / 2}$ \\
push_particle_p(prt); & $/ / \mathbf{p}^{n} \rightarrow \mathbf{p}^{n+1}$ \\
push_particle_x(prt); & $/ /$ save $\mathbf{x}^{n+1 / 2}$ for current, then $\mathbf{x}^{n+1 / 2} \rightarrow \mathbf{x}^{n+3 / 2}$ \\
deposit_j(); & $/ /$ charge conservative current deposition using $\mathbf{x}^{n+1 / 2}$ and $\mathbf{x}^{n+3 / 2}$ \\
f & \\
push_field_B_half ()$;$ & $/ / \mathbf{B}^{n+1 / 2} \rightarrow \mathbf{B}^{n+1}$ \\
exchange_particles ()$;$ & $/ /$ communicate \\
fill_ghosts_B(); & $/ /$ communicate \\
$\begin{array}{l}\text { add_and_fill_ghosts_j(); } \\
\text { push_field_E(); }\end{array}$ & $/ / / \mathbf{E}^{n+1 / 2} \rightarrow \mathbf{E}^{n+3 / 2}$ \\
\hline
\end{tabular}

Figure 8. Optimized implementation of the timestep in Psc. It advances $\mathbf{B}^{n}, \mathbf{p}^{n} \rightarrow \mathbf{B}^{n+1}, \mathbf{p}^{n+1}$ and $\mathbf{E}^{n+1 / 2}, \mathbf{x}^{n+1 / 2} \rightarrow \mathbf{E}^{n+3 / 2}, \mathbf{x}^{n+3 / 2}$ and performs all communication at a single synchronization point.

case, so this was still a large improvement. It does, however, show that as we balance the particle load the field load becomes imbalanced and creates a new loss of performance that manifests itself in the overall timestep slow-down.

With careful consideration, it is possible to improve balancing to include both particle and field work. The basis for the updated load balancing is to rewrite the time step closer to the natural time-staggered form in our numerical algorithms. In the new algorithm, we start a time step with the quantities known as $\mathbf{E}^{n+1 / 2}, \mathbf{B}^{n}, \mathbf{x}^{n+1 / 2}, \mathbf{p}^{n}$, and propagate them to $\mathbf{E}^{n+3 / 2}, \mathbf{B}^{n+1}, \mathbf{x}^{n+3 / 2}, \mathbf{p}^{n+1}$ as shown in Fig. 8 .

Every quantity is now updated only once, by a full step, with the exception of $\mathbf{B}^{n} \rightarrow \mathbf{B}^{n+1 / 2} \rightarrow \mathbf{B}^{n+1}$, which is required at the intermediate time to interpolate the Lorentz force acting on particles.

Other than the drawback of having to handle quantities at different time levels at the initial condition and output, the scheme in its natural form presents a number of advantages: Less computational work is required due to combining half steps into full steps. The discrete version of Gauss's Law is required to be satisfied exactly at half-integer time levels, which can now be achieved more easily in the initial condition. Particles are exchanged according to their positions at time $t^{n+1 / 2}$, so particles are guaranteed to actually be inside the local domain at the time that the electromagnetic fields are interpolated to the particle position. This is in contrast to the old scheme, where a particle already moved a half time step, and hence might have left the local domain. This means that fewer levels of ghost points are required.

Most importantly, the rewritten scheme can be recast to have only a single synchronization point. We now do all communication after the particle push and after completing the second half step to update $\mathbf{B}$. At that point, particles are ready to be exchanged as their positions have been advanced to $\mathbf{x}^{n+3 / 2}$. The current density $\mathbf{j}^{n+1}$ has been calculated and can be added up and used to fill ghost points. After we also fill ghost points for $\mathbf{B}^{n+1}$, enough information is available to perform the remaining field updates all the way to the next particle push, while still providing the necessary ghost cell data for the field interpolations in the next particle push.

For second-order particle shape functions, two layers of ghost points for the fields $\mathbf{j}, \mathbf{E}$, and $\mathbf{B}$ are sufficient to perform a full time step, including field and particle updates, without any further communication.

\subsubsection{Calculating the load function}

As the time integration now requires only a single synchronization point, it is possible to balance the total computational load per timestep, including both particle and field updates. The load balancing algorithm requires as input an estimate of the load $L_{p}$ associated with the computations occuring on each of the patches, $p$. A promising candidate is a function of the form

$$
L_{p}=N_{\text {particles }}(p)+C N_{\text {cells }}(p),
$$

as the work in the particle push scales with the number of particles being pushed, while the field updates scale with the number of grid cells. The constant $C$ can be used to adjust the weighting between particle push and field updates, as the work of pushing one particle is not expected to be equal to the work of advancing the fields in one cell; we find values of order 1 to work well. As we will show in a case study later, this simple approximation works quite well to 


\begin{tabular}{ccc}
\hline \hline Global number of patches & Patches per process & Patch size \\
\hline $30 \times 20$ & 1 & $40 \times 40$ \\
$60 \times 40$ & 4 & $20 \times 20$ \\
$120 \times 80$ & 16 & $10 \times 10$ \\
$150 \times 100$ & 25 & $8 \times 8$ \\
$240 \times 160$ & 64 & $5 \times 5$ \\
\hline \hline
\end{tabular}

Table 2. List of runs to study the performance cost of dividing the domain into many small patches.

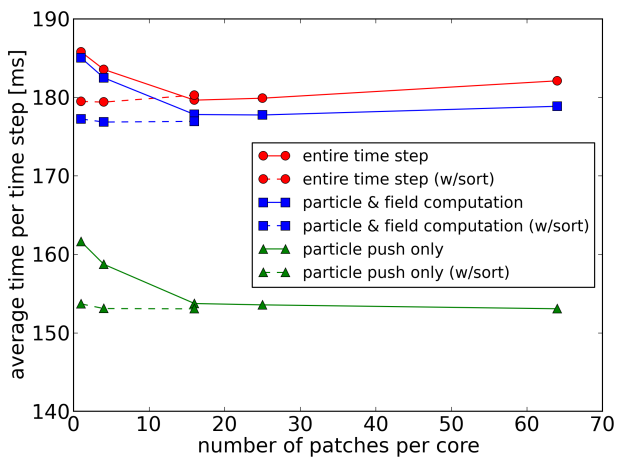

Figure 9. Timing data for naturally balanced runs while varying the number of patches that the domain is decomposed into from 1 patch per core to 64 patches per core.

achieve good balance. It requires, however, an appropriate choice of the parameter $C$, so we also pursued an alternate approach of actually measuring the time spent in the computational kernels.

\subsubsection{Performance cost of subdividing the domain}

Subdividing the spatial domain into many more patches than processing units can improve the load balance of a particle-in-cell simulation dramatically. On the other hand, besides the increased complexity in implementing the approach, there are also potential performance costs: (1) Rebalancing the domain, including moving patches to other processes in order to improve load balance takes processing time. (2) Handling many small patches on a process rather than just one large patch creates costs in managing those patches, in increased computational work, and in increased communication both between local patches and between local and remote patches.

While the cost of rebalancing (1) is substantial, typically equal to a couple of regular timesteps, rebalancing only needs to be performed occasionally, typically every $100-500$ steps, so this cost gets amortized over a large number of steps. As will be shown in a case study below, we find that the cost of rebalancing only adds an amortized cost of 1$2 \%$. Rebalancing involves one global gather operation that collects the loads for all patches; the new load distribution is redundantly calculated from that on each processor, and communication is initiated to move actual particle and field data for patches that are moved to a different process. As the shifting occurs along the one-dimensional spacefilling curves, the data communication occurs point-to-point between a MPI process and typically only its nearest 1-d neighbors, though drastic changes in the loads can involve a few close neighbors.

In order to address issue (2), we performed a number of simulations at identical physical and numerical parameters, while varying the number of patches that the domain is divided into. We used physical plasma parameters motivated by the bubble reconnection simulations that will be described in more detail later, but changed the initial condition to be a uniform plasma. Using a bubble simulation directly is not feasible, since the initial density is non-uniform and, even if the simulation is initially load balanced, it quickly becomes unbalanced. The initially uniform plasma remains uniform, which means that no actual load balancing is required and we can exclude the impact of growing imbalance and focus just on the peformance cost of varying the partitioning into patches. Our example case is run at a resolution of $1200 \times 800$ grid cells using 600 cores, using 200 particles per cell per species. We start with a simple decomposition into $30 \times 20$ patches, which means that every process handles only a single patch of size $40 \times 40$ grid cells. In this case 
there is no additional cost from subdividing the domain into smaller patches, it is just standard domain decomposition. We then increase the number of patches the domain is divided into progressively up to $240 \times 160$, i.e. 64 patches per process of size $5 \times 5$ each. Table 2 lists the parameters for the simulations we performed.

Fig. 9 plots average performance data vs the number of patches per MPI process. The first thing to notice is that the variation of the total time per time step (red curve with circles) is fairly small: it varies between $179.4 \mathrm{~ms}$ and $185.8 \mathrm{~ms}$, i.e., the slowest case is less than $4 \%$ slower than the fastest. The plotted data were obtained by averaging the timing measurements over simulation time steps 500 to 1000 (we skipped the initial 500 steps in order to avoid measuring transients from the initial condition.) At each time step, Psc measures the wallclock time to perform certain work, and then finds minimum, maximum and average over all MPI processes. We show the maximum values in the plot, as the slowest processes generally determine overall performance, though there is little variation between minimum, maximum and average as the simulation is naturally balanced.

The total time per time step initially goes down (solid red curve) as the number of patches is increased up to 16 patches per process, and then goes up again. This might seem surprising, but is easily explained by the limited cache memory available to each core. As the patch size decreases to $10 \times 10$, field data is more effectively cached - all particles in any one patch are processed before moving on to the next patch, so the fields will quickly become resident in cache, allowing to push all particles in that patch without further access to field data in main memory. We confirm this by looking at the total computational work per timestep (blue curve with squares) and the particle push time (green curve with triangles). It is clear that the faster total time per time step originates in the particle pusher. The clearest evidence comes from re-running the simulations with particle sorting enabled every 50 steps (dashed lines). Since particles are now always sorted, fields are accessed in a structured manner independent of the patch size, and we consistently see fast performance. With sorting enabled, performance is now in fact fastest when using only one patch per processor, avoiding the additional overhead of multiple patches. However, the performance cost of using many patches is very small. Even at 64 patches / core, which corresponds to a very small patch size of $5 \times 5$ grid cells, the time step is only $1.5 \%$ slower than in the fastest case. This performance loss, small as it is, can be traced down to two causes: (1) The total computational work per timestep (blue curve) increases - this is mainly caused by the additional ghost cells that Maxwell's equations are solved in (as we laid out before, some ghost cell values are calculated rather than communicated to avoid additional communication and synchronization points). (2) The time spent exchanging particles and ghost cell values increases. This time can be seen in the figure as the difference between the blue and the red curve, and it clearly increases as the number of patches per core increases. Most of the additional communication occurs between patches on the same MPI process, where it is handled by simple copies, rather than actual message passing, which keeps its overall impact small.

From the data we presented here, it is clear that the overhead of using many patches per process remains quite small as long as the patch size is not made unreasonably small.

\subsection{Performance study: Load balancing a bubble reconnection simulation}

Our first case for studying the utility and performance of the space-filling curve based load balancing scheme in PSC is a simulation of magnetic reconnection of laser-produced plasma bubbles. More detail about those simulations and the underlying physics can be found in $[10,11]$. The runs presented below used a background plasma density of 0.05 and a peak density of 1.0 in the center of the bubbles in normalized units. The plasma bubbles expand into each other, driving magnetic reconnection; in the process, the peak density moves from the bubble center to the edge. We used a mass ratio of $m_{i} / m_{e}=100$. The domain size is $60 d_{i} \times 40 d_{i}$, we use $2400 \times 1600$ grid cells. All runs were performed using 2048 cores of the Cray XE6m supercomputer Trillian located at the University of New Hampshire. We will compare 4 approaches to domain decomposition and load balancing: (1) uniform decomposition, (2) static rectilinear decomposition, (3) dynamic rectilinear decomposition, and (4) dynamic patch-based decomposition.

\subsubsection{Uniform decomposition}

This easiest approach, i.e. dividing the domain into as many equal-sized subdomains as there are processors (see Fig. 3), does not afford any opportunity to balance the computational load, which ends up being substantially unbalanced at a factor of $11 \times$. This actually made it infeasible to run a complete simulation, and the timing was so far off compared to the other methods that we chose to scale Fig. 10 to focus on the results from the more promising load balancing methods. 


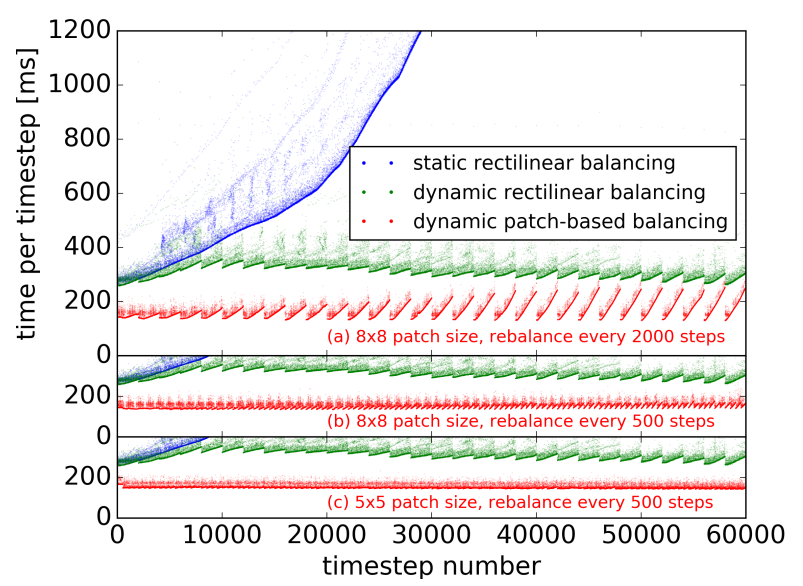

Figure 10. Wall clock time per timestep for runs with static rectilinear (blue), dynamic rectilinear (green), and dynamic patch-based (red) load balancing.

\subsubsection{Rectilinear balancing}

As mentioned earlier, PSC originally supported what we call "static rectilinear load balancing". That is, at initial set up time the decomposition boundaries are shifted along the coordinate axes to achieve better load distribution as shown in Fig. 11 (top). This decomposition substantially improves load balance - initial imbalance is now down to less than $2 \times$. Fig. 10 compares rectilinear load balancing to our new patch-based approach, showing the evolution of the compute time per timestep. The statically balanced case (blue) starts out $1.7 \times$ slower than the patch-based approach, but load balance quickly increases. As originally implemented, there was no dynamic rebalancing, so the slow-down was drastic once the bubble dynamics substantially changed the plasma configuration, and we did not continue the solution past the halfway point.

We added the capability to dynamically rebalance the rectilinear approach, which is shown by the green curve. The effect of rebalancing every 2000 steps can be clearly seen - imbalance still grows initially, but is then arrested and is remains under $2.5 \times$. While not ideal, this allowed us to perform these kind of simulations to completion.

\subsubsection{Patch-based balancing}

The resulting decompositions from using the patch-based approach are shown in Fig. 12. The figures shows that the per-MPI process subdomains (demarcated by black lines) are indeed well clustered in space by means of using the space-filling curve. Local subdomains range from encompassing many ( 100) low-density patches down to just 4 of the highest density patches. As shown in red on Fig. 10, we achieve rather good load balance using this new method and are able to maintain it in time given the right parameters.

Fig. 10 compares three different runs. (a) used patches of size $8 \times 8$ grid cells, rebalancing every 2000 steps. It can be seen that at later times the load quickly becomes unbalanced again after each rebalancing step, so better average balance is obtained using more frequent rebalancing as shown in (b) at every 500 steps. Finally, in (c) we used more, even smaller patches of size of $5 \times 5$ which, in fact, still worked very well. As expected the load balance improves a bit, while the increased overhead does not substantially affect performance.

\subsubsection{Timing comparison}

The clearest indication of how well our new load balancing method works is given by comparing overall timing of the runs. In Table 3, we list overall timing information, comparing uniform, static and dynamic rectilinear, and patch-based balancing. Data are normalized by the performance of the new patch-based approach, i.e. we show the slow-down compared to this method. As previously mentioned, the uniform (not balanced) approach is very expensive for our test case, so we did not run a simulation beyond an initial phase where it was almost $10 \times$ slower than the patch-based method. The rectilinear approaches do quite well initially, with only a slowdown of $1.7 \times$. Static balancing, however, is not sufficient: The simulation down to 30,000 steps was 3.6× slower and rapidly degraded even 

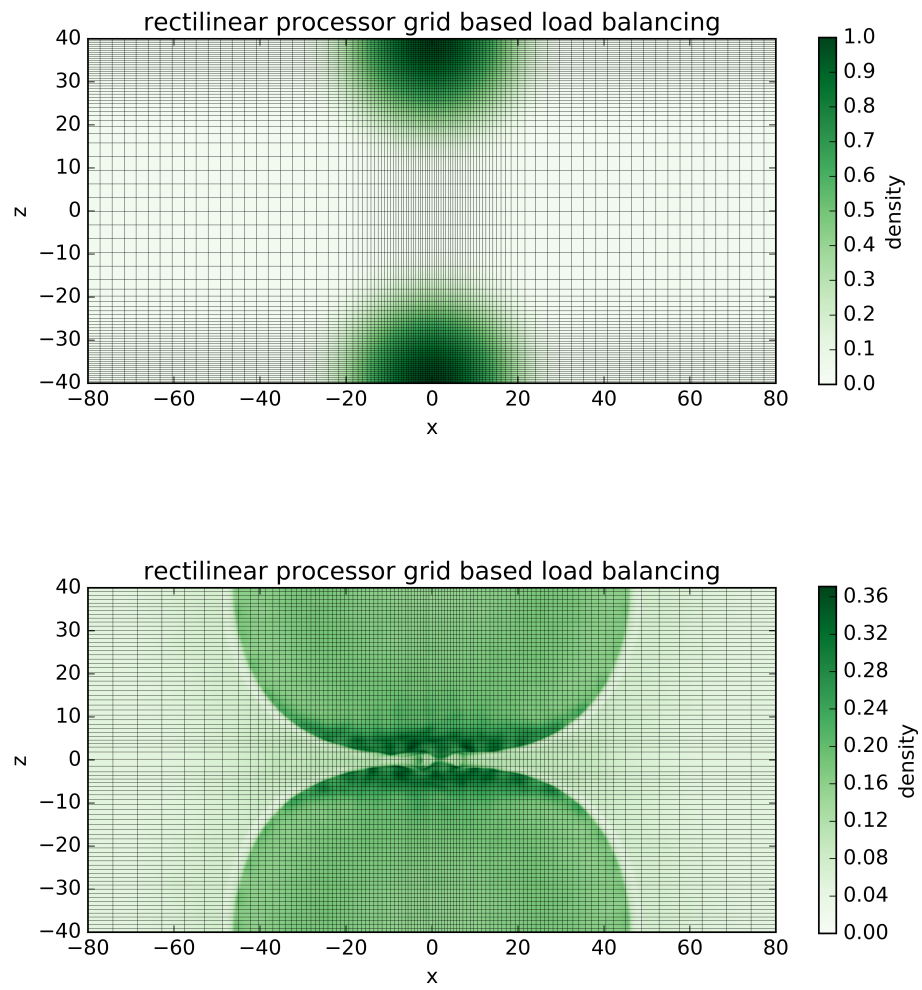

Figure 11. Rectilinar decomposition by shifting decomposition boundaries. (top) at the initial time, (bottom) shifted decomposition after time of maximum reconnection rate at 50,000 steps. The plasma density is shown in green.

\begin{tabular}{crrr}
\hline \hline balancing method & initial & first 30,000 steps & all 60,000 steps \\
\hline uniform & $9.9 \times$ & $\mathrm{n} / \mathrm{a}$ & $\mathrm{n} / \mathrm{a}$ \\
static rectilinear & $1.66 \times$ & $3.64 \times$ & $\mathrm{n} / \mathrm{a}$ \\
dynamic rectilinear & $1.66 \times$ & $1.94 \times$ & $1.87 \times$ \\
dynamic patch-based & $1 \times$ & $1 \times$ & $1 \times$ \\
\hline \hline
\end{tabular}

Table 3. Slow-down of various load balancing methods compared to dynamic patch-based method. 

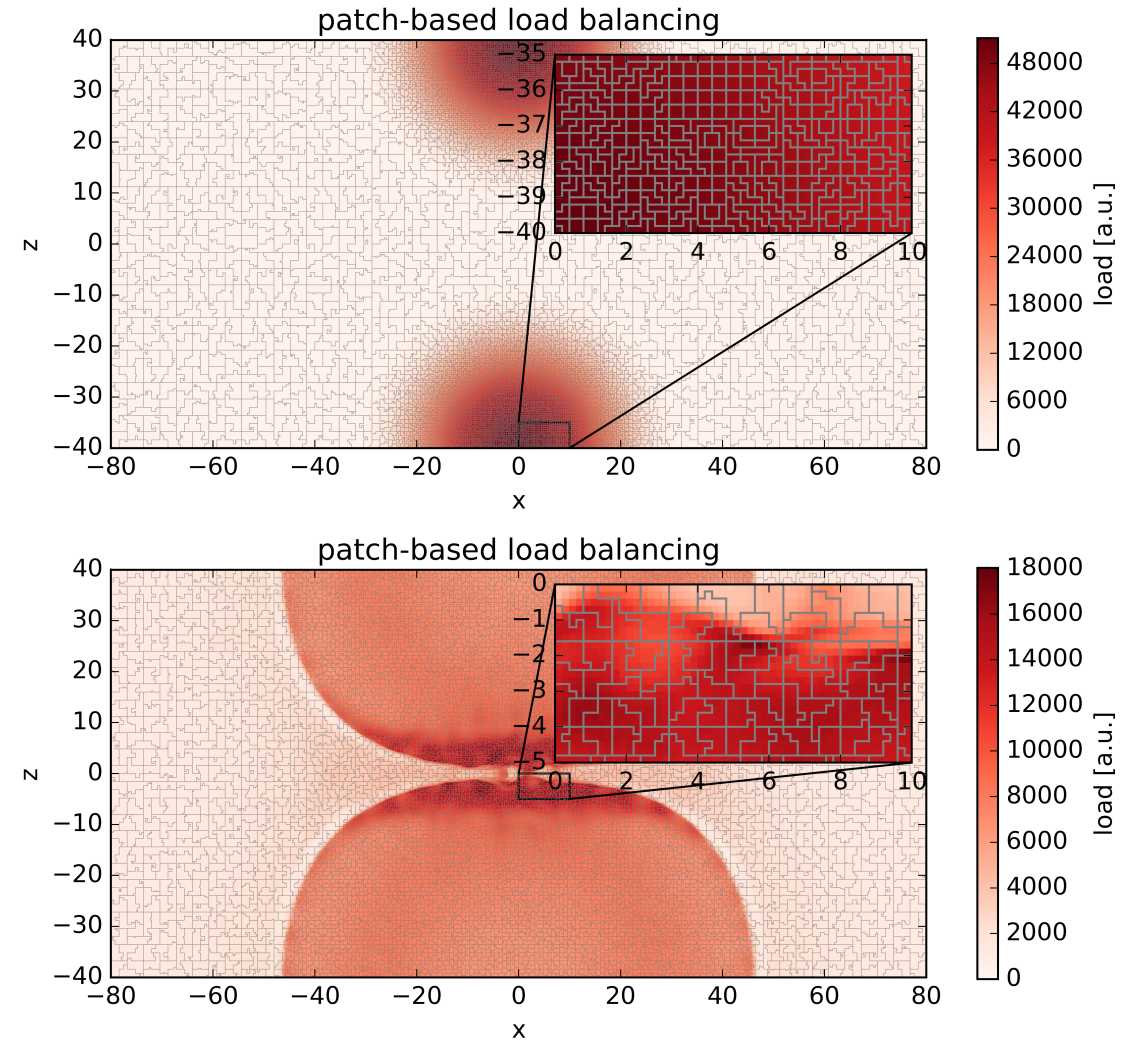

Figure 12. Patch-based load balancing. (top) at the initial time, (bottom) shifted decomposition after time of maximum reconnection rate at 50,000 steps. Show in red is the actual load per patch, which follows the number of particles and plasma density, and the decomposition boundaries (black). 
more, so that we did not continue it all the way to the end. The dynamic rectilinear approach performed reasonably well, with the simulation running not quite two times longer than the new approach.

Overall, we showed that our new patch-based load balancing approach works very well and is in fact clearly superior over the alternate methods that previously existed in PSC.

\subsubsection{Estimating the load}

We used this test case to also compare different methods of estimating the computational load per patch, which is needed as input to the load balancing mechanism. We tried formula Eq. (36) with $C=1$ and $C=2$ and also compared it to a real time load estimator that instead of using an analytic formula actually measured timing data as the simulation proceeded. We did not observe any significant differences in the achieved balance, though we noticed that the timing data was somewhat noisy so the achieved load balance in that case would bounce around to some small extent.

\subsection{Performance study: Load balancing a particle acceleration study}

We performed another performance study on a simulation of particle acceleration in magnetic reconnection, to show that load imbalance can be significant in a more common application, too. The simulation parameters used here are motivated by the work in [3]. The initial condition is a Harris sheet with a central density of $n=1$ and a background density of $n=0.05$. The domain size is $204.8 d_{i} \times 51.2 d_{i}=4096 d_{e} \times 1024 d_{e}$ using a $16384 \times 4096$ cell mesh. The mass ratio is $m_{i} / m_{e}=400$. We used 200 particles per cell to represent $n=1$ and the 2 nd order particle shape function.

This simulation poses substantial challenges for maintaining load balance. Initially the plasma density away from the current sheet is $20 \times$ lower than in the central current sheet, shown in Fig. 13(a). Consequently the load per patch is similarly unbalanced, varying from 1344 to 26592 in the center, a ratio of 19.8. The simulation divides the domain into $1024 \times 256=262144$ patches of $8 \times 8$ cells, and is run on 8192 cores. This averages to 32 patches / core, but due to the strong imbalance the achieved load balance is only within $\pm 32 \%$ - still a vast improvement over the original $20 \times$.

As the simulation proceeds load balancing becomes even more challenging since reconnection occurs, some plasmoids form and get ejected, and almost all of the density gets concentrated in few islands while the plasma in the rest of the domain becomes very tenuous.

After 70000 steps (see Fig. 13(b)), the load varies from a minimum of 228 to a maximum of 52011, an imbalance of $230 \times$. The code handles the increasing load balance quite well, though, balancing process load to within $\pm 50 \%$.

Fig. 14 shows the timing information throughout the run. The black curve again indicates the execution time of an entire timestep - it only increases mildly at around 50000 steps, which is when plasmoids start forming, and then remains approximately flat again thereafter. We again see that the black line is only slightly above the blue line, the computational time used on the process with the heaviest load. This indicates that communication / boundary exchange is not a significant performance factor. The spread of the green (fastest) and blue (slowest) process from the average (red) indicates that load balance is not perfect, in fact these mirror the aforementioned $\pm 32 \%, \pm 50 \%$ deviation from perfect balance. This spread is certainly acceptable considering that the underlying imbalance is greater than 200×. The faint black dots at around $300 \mathrm{~ms}$ are slower time steps that include rebalancing - these occur every 500 steps and have a noticable cost, but don't affect the overall performance significantly.

\section{Conclusions}

In this paper, we have described the numerical and computational methods used in the explicit electromagnetic particle-in-cell code PSc.

It can make use of the capabilities of today's massively parallel super-computers and can be run on 10,000's or 100,000 's of conventional cores. PSc's flexible design allows for changing algorithms and data structures to support current and future processor designs. As an example, Fig. 15 characterizes Psc's overall scalability, comparing CPUonly to GPU-enabled runs for the two-dimensional Kelvin-Helmholtz instability. This weak scaling study shows that parallel efficiency is maintained at better than $90 \%$ scaling up from 20 GPUs to 5120 GPUs, or correspondingly scaling from 320 CPU cores to 81920 CPU cores on ORNL's Titan machine. While we leave the details of PSC's GPU 
(a)

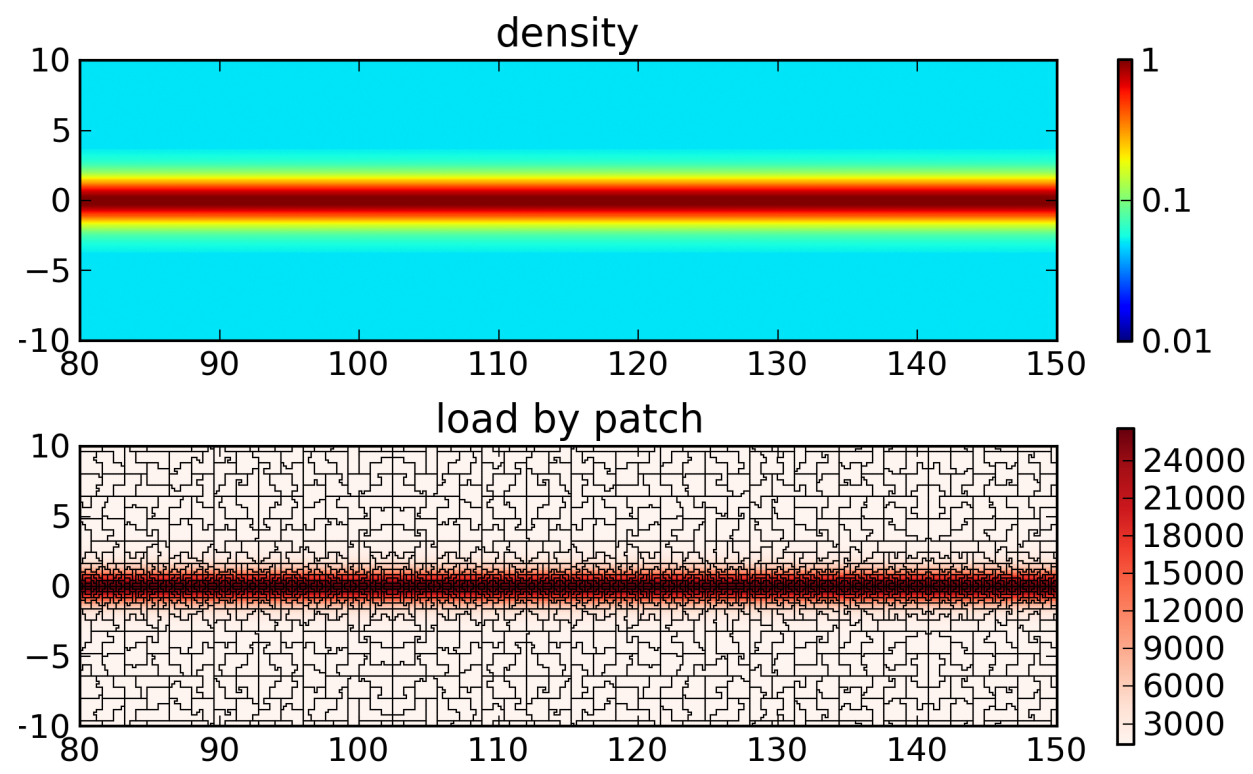

(b)

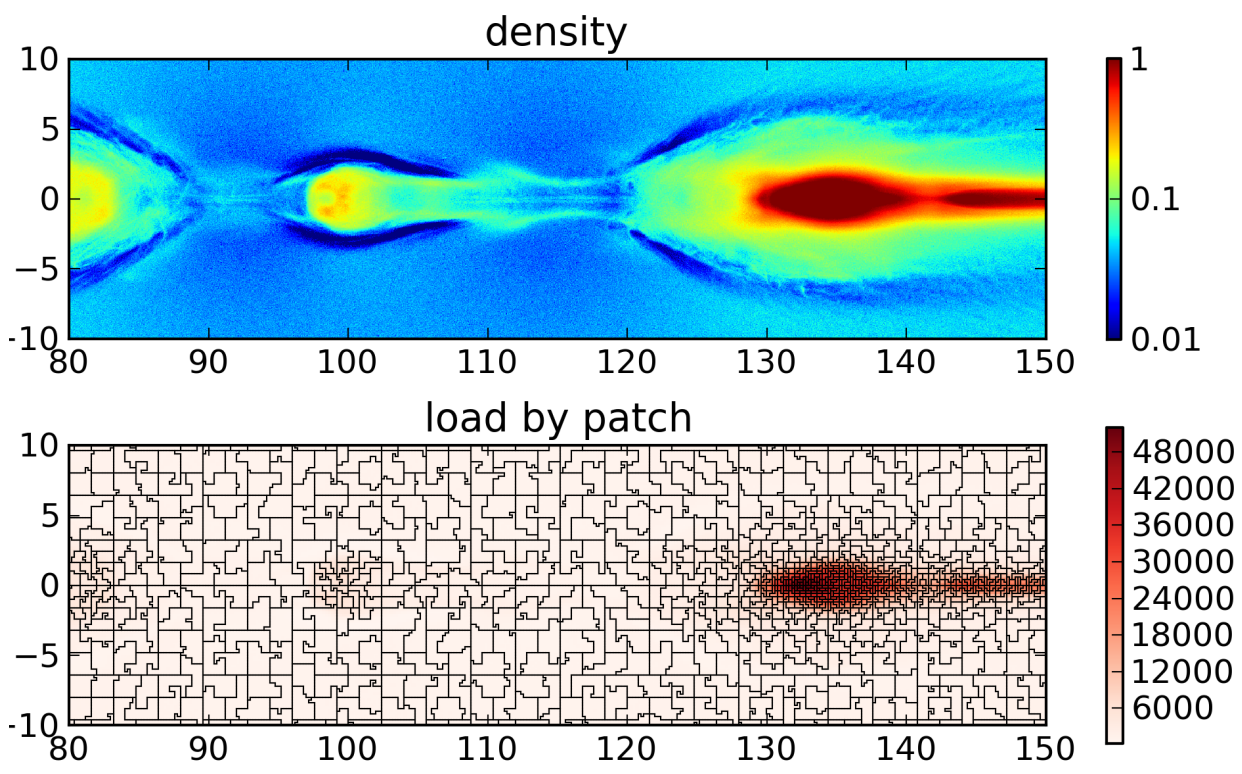

Figure 13. (a) Initial Harris sheet and (b) reconnection in progress after 70000 steps. Shown are the density on a logarithmic scale (top), and the load for each patch and the subdomain boundaries (bottom). Show is a zoom into the central region of the current sheet. 


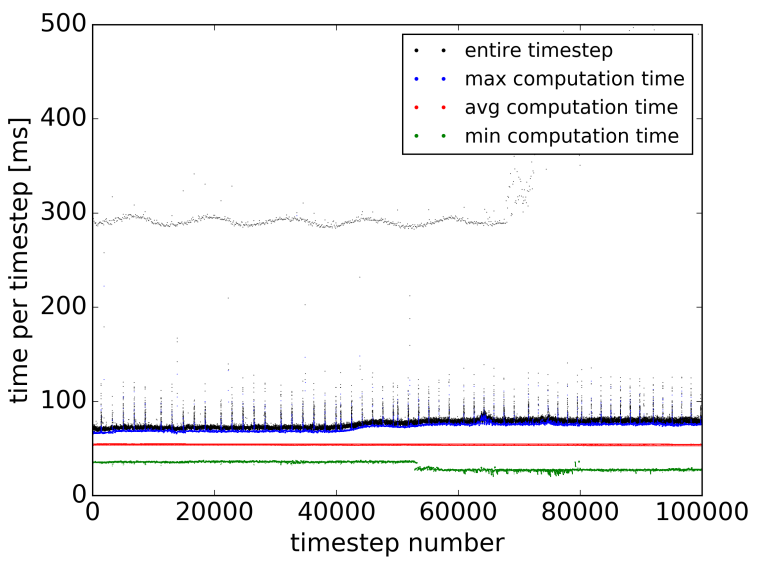

Figure 14. Timing data for the particle acceleration run, with dynamic load balancing performed every 100 timesteps. The execution time for the entire timestep is plotted in black. The time spent in actual computation is shown in blue, red and green for the slowest, average and fastest process, respectively

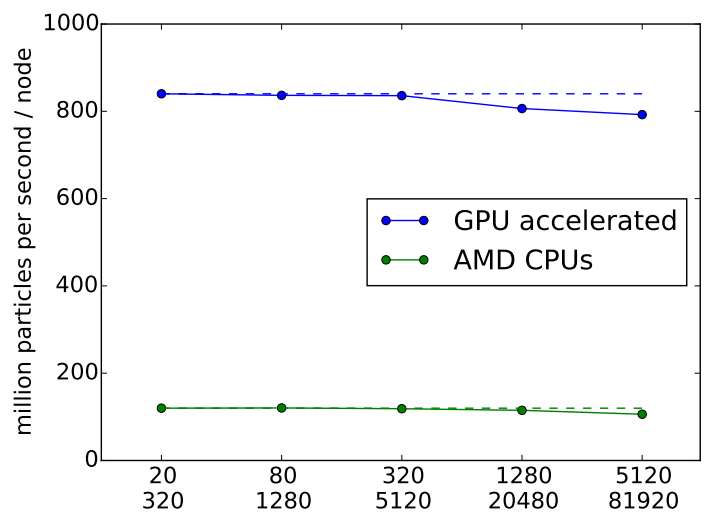

top: number of Cray XK nodes (16-core AMD CPU + 1 Tesla 20X GPU) bottom: number of Cray XK AMD CPU cores

Figure 15. Weak scaling study of Psc on Titan, using from 20 to 5120 16-core nodes with one GPU each, and correspondingly to 320 to 81920 CPU cores for CPU-only runs. 
support for a separate paper, the plot not only demonstrates good parallel scalability, but also how the same code can run on CPUs or GPUs depending on command line options, and achieve a significant speed-up of over $6 \times$ by using the GPUs.

We have described and analyzed in detail a distinguishing features of PSc:, support for patch-based dynamic load-balancing using space-filling curves, which effectively addresses performance issues in simulations where large numbers of particles move to different regions of the spatial domain, causing imbalance of the computational load on the decomposed domain. Using two case studies, we have shown that Psc's new load balancing method achieves and maintains good computational performance over the entire run.

PSC encompasses both production and development capabilities in one code, allowing for state-of-the-art science simulations while at the same time, experimental new features are being developed. For example, while we are working on stabilizing 3-d GPU support and getting it production-ready, another effort is underway to implement support for Intel's new many-integrated-cores processor technology (Intel Xeon Phi) which takes advantage of their 512-bit SIMD capabilities. It remains unclear where exactly the path of high-performance computation on the way to the exascale leads, but it is fairly clear that it will involve specialized number-crunching hardware, rather than just conventional cores. So far, the approach that we have taken with PSc is to use hardware-specific programming models, e.g., CUDA on GPUs or SIMD intrinsics on Intel MIC. Since only the most performance-intensive kernels need to be ported, this approach is manageable but still work-intensive. In the future, we hope to be able to leverage abstracted programming models like OpenACC/OpenMP, which fit well into the existing modular design of the code.

\section{Acknowledgements}

This research was supported by DOE grants DE-SC0006670 and DE-FG02-07ER46372, NSF grants AGS-105689 and and NASA grant NNX13AK31G.

Computational work has been performed on DOE's Titan machine at ORNL and NERSC systems, and the Cray XE6m Trillian at the University of New Hampshire, funded with support from NSF's MRI program under PHY1229408. An award of computer time was provided by the Innovative and Novel Computational Impact on Theory and Experiment (INCITE) program. This research used resources of the Oak Ridge Leadership Computing Facility, which is a DOE Office of Science User Facility supported under Contract DE-AC05-00OR22725.

The authors acknowledge helpful discussions with Homa Karimabadi, Bill Daughton and Vadim Roytershteyn.

\section{Appendix A. Load balancing algorithm}

The load balancing algorithm takes as input an array of the loads for each patch $L$, and a list of capabilities for each process rank $C_{r}$. The output is an array of the patch count $N_{r}$ that each process rank is assigned. 


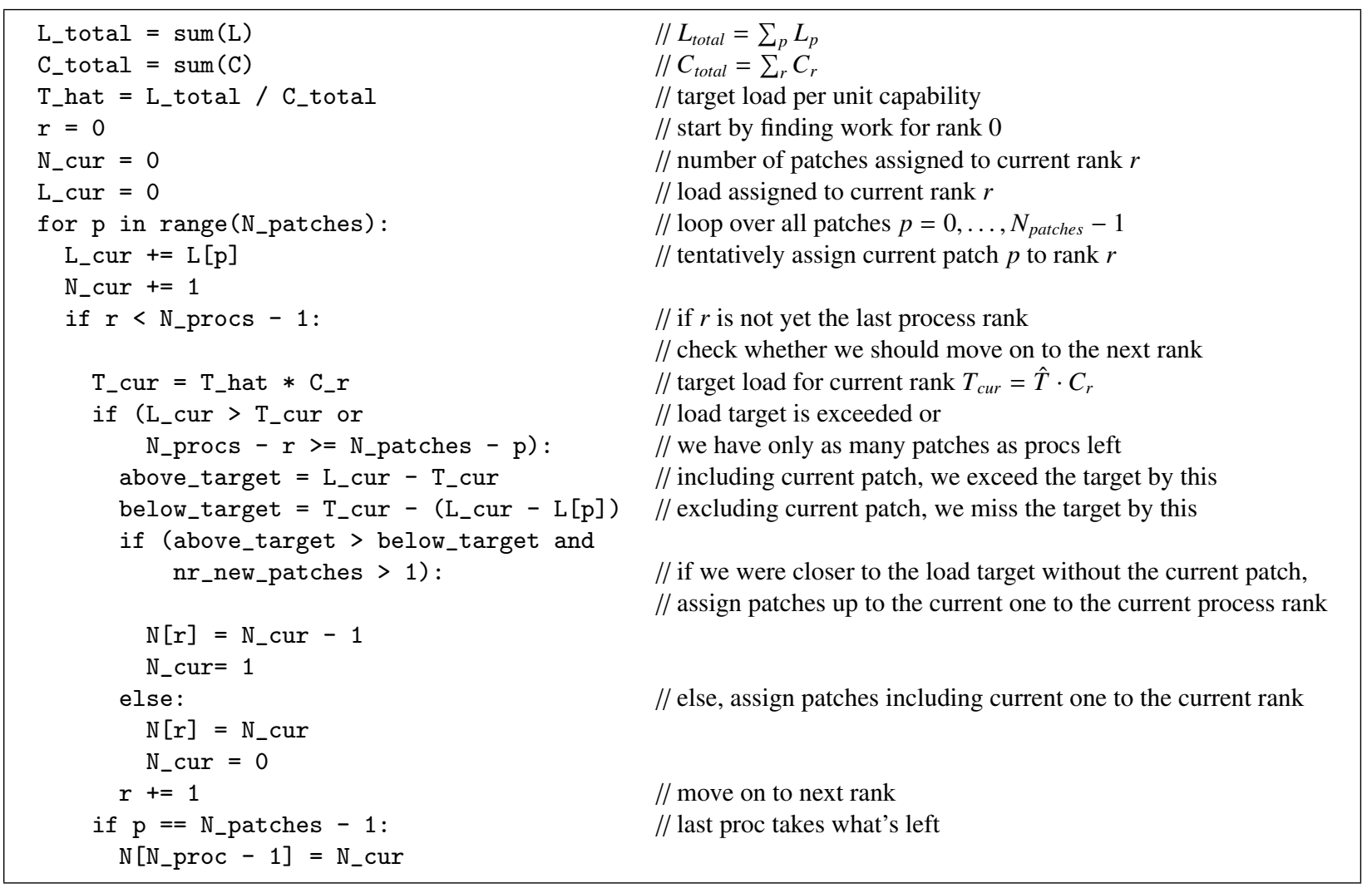

\section{References}

[1] J. Egedal, A. Le, W. Daughton, A review of pressure anisotropy caused by electron trapping in collisionless plasma, and its implications for magnetic reconnection, Physics of Plasmas (1994-present) 20 (2013)

[2] H. Schmitz, R. Grauer, Comparison of time splitting and backsubstitution methods for integrating Vlasov's equation with magnetic fields, Computer Physics Communications 175 (2006) 86-92.

[3] J. Egedal, W. Daughton, A. Le, Large-scale electron acceleration by parallel electric fields during magnetic reconnection, Nature Physics (2012).

[4] L. Chacón, G. Chen, D. Barnes, A charge- and energy-conserving implicit, electrostatic particle-in-cell algorithm on mapped computational meshes, Journal of Computational Physics 233 (2013) $1-9$.

[5] G. Lapenta, Particle simulations of space weather, Journal of Computational Physics 231 (2012) 795 - 821.

[6] K. J. Bowers, B. J. Albright, L. Yin, B. Bergen, T. J. T. Kwan, Ultrahigh performance three-dimensional electromagnetic relativistic kinetic plasma simulation, Physics of Plasmas 15 (2008) 055703.

[7] R. A. Fonseca, L. O. Silva, F. Tsung, V. K. Decyk, W. Lu, C. Ren, W. B. Mori, S. Deng, S. Lee, T. Katsouleas, et al., Osiris: A threedimensional, fully relativistic particle in cell code for modeling plasma based accelerators, in: Computational Science-ICCS 2002, Springer, 2002, pp. 342-351.

[8] C. Nieter, J. R. Cary, Vorpal: a versatile plasma simulation code, Journal of Computational Physics 196 (2004) $448-473$.

[9] H. Ruhl, Classical particle simulations, in: M. Bonitz, D. Semkat (Eds.), Introduction to Computational Methods in Many Body Physics, Rinton Press, 2006.

[10] W. Fox, A. Bhattacharjee, K. Germaschewski, Fast magnetic reconnection in laser-produced plasma bubbles, Phys. Rev. Lett. 106 (2011) 215003.

[11] W. Fox, A. Bhattacharjee, K. Germaschewski, Magnetic reconnection in high-energy-density laser-produced plasmas, Physics of Plasmas 19 (2012) 056309.

[12] W. Fox, G. Fiksel, A. Bhattacharjee, P.-Y. Chang, K. Germaschewski, S. X. Hu, P. M. Nilson, Filamentation instability of counterstreaming laser-driven plasmas, Phys. Rev. Lett. 111 (2013) 225002.

[13] G. Fiksel, W. Fox, A. Bhattacharjee, D. H. Barnak, P.-Y. Chang, K. Germaschewski, S. X. Hu, P. M. Nilson, Magnetic reconnection between colliding magnetized laser-produced plasma plumes, Phys. Rev. Lett. 113 (2014) 105003

[14] N. Bessho, L.-J. Chen, K. Germaschewski, A. Bhattacharjee, Electron acceleration by parallel and perpendicular electric fields during magnetic reconnection without guide field, Journal of Geophysical Research: Space Physics (2015). 2015 JA021548. 
[15] L. Wang, A. H. Hakim, A. Bhattacharjee, K. Germaschewski, Comparison of multi-fluid moment models with particle-in-cell simulations of collisionless magnetic reconnection, Physics of Plasmas 22 (2015).

[16] C. Birdsall, A. Langdon, Plasma Physics Via Computer Simulation, Series in plasma physics, Taylor \& Francis, 1991.

[17] R. Hockney, J. Eastwood, Computer Simulation Using Particles, McGraw-Hill, 1981.

[18] L. Greengard, V. Rokhlin, A fast algorithm for particle simulations, Journal of Computational Physics 135 (1997) 280 - 292.

[19] K. Yee, Numerical solution of initial boundary value problems involving maxwell's equations in isotropic media, Antennas and Propagation, IEEE Transactions on 14 (1966) 302-307.

[20] J. Boris, Acceleration calculation from a scalar potential, 1970.

[21] J. Villasenor, O. Buneman, Rigorous charge conservation for local electromagnetic field solvers, Computer Physics Communications 69 (1992) 306-316.

[22] T. Esirkepov, Exact charge conservation scheme for particle-in-cell simulation with an arbitrary form-factor, Computer Physics Communications 135 (2001) $144-153$

[23] I. V. Sokolov, Alternating-order interpolation in a charge-conserving scheme for particle-in-cell simulations, Computer Physics Communications 184 (2013) $320-328$

[24] E. Evstatiev, B. Shadwick, Variational formulation of particle algorithms for kinetic plasma simulations, Journal of Computational Physics 245 (2013) $376-398$

[25] A. B. Langdon, On enforcing Gauss' law in electromagnetic particle-in-cell codes, Computer Physics Communications 70 (1992) 447-450.

[26] T. Takizuka, H. Abe, A binary collision model for plasma simulation with a particle code, Journal of Computational Physics 25 (1977) 205-219.

[27] M. E. Jones, D. S. Lemons, R. J. Mason, V. A. Thomas, D. Winske, A grid-based coulomb collision model for $\{$ PIC $\}$ codes, Journal of Computational Physics 123 (1996) $169-181$.

[28] A. J. Kemp, H. Ruhl, Multispecies ion acceleration off laser-irradiated water droplets, Physics of Plasmas 12 (2005) 033105.

[29] W. Daughton, J. Scudder, H. Karimabadi, Fully kinetic simulations of undriven magnetic reconnection with open boundary conditions, Physics of Plasmas 13 (2006).

[30] A. Bhattacharjee, K. Germaschewski, C. Ng, Current singularities: Drivers of impulsive reconnection, Phys. Plasmas 12 (2005) 042305.

[31] K. Germaschewski, A. Bhattacharjee, C. Ng, The magnetic reconnection code: an amr-based fully implicit simulation suite, in: N. Pogorelov, G. Zank (Eds.), Numerical Modeling of Space Plasma Flows: Astronum-2006, volume 359, ASP Conference Series, 2007 , p. 15.

[32] K. Germaschewski, J. Raeder, D. J. Larson, A. Bhattacharjee, New developments in modeling MHD systems on high performance computing architectures, in: N. Pogorelov, G. Zank (Eds.), Numerical Modeling of Space Plasma Flows: Astronum-2008, volume 406, ASP Conference Series, 2009, pp. 223-230.

[33] J. Raeder, Global Magnetohydrodynamics - A Tutorial, in: J. Büchner, C. T. Dum, M. Scholer (Eds.), Space Plasma Simulation, Springer Verlag, Berlin Heidelberg New York, 2003, pp. 212-246.

[34] K. Germaschewski, J. Raeder, Using automated code generation to support high performance extended mhd integration in openggcm, in: N. Pogorelov, G. Zank (Eds.), Numerical Modeling of Space Plasma Flows: Astronum-2010, volume 444, ASP Conference Series, 2011 , p. 197.

[35] K. Germaschewski, GitHub repositories for libmrc and psc, 2014. https://github.com/germasch.

[36] S. Balay, K. Buschelman, W. D. Gropp, D. Kaushik, M. G. Knepley, L. C. McInnes, B. F. Smith, H. Zhang, PETSc Web page, 2001. http://www.mcs.anl.gov/petsc.

[37] S. Balay, K. Buschelman, V. Eijkhout, W. D. Gropp, D. Kaushik, M. G. Knepley, L. C. McInnes, B. F. Smith, H. Zhang, PETSc Users Manual, Technical Report ANL-95/11 - Revision 2.1.5, Argonne National Laboratory, 2004.

[38] S. Balay, W. D. Gropp, L. C. McInnes, B. F. Smith, Efficient management of parallelism in object oriented numerical software libraries, in: E. Arge, A. M. Bruaset, H. P. Langtangen (Eds.), Modern Software Tools in Scientific Computing, Birkhäuser Press, 1997, pp. 163-202.

[39] T. H. Group, Hierarchical data format version 5, 2000-2010.al data format version 5, http://www . hdf group.org/HDF5, 2000-2010.

[40] H. Burau, R. Widera, W. Hönig, G. Juckeland, A. Debus, T. Kluge, U. Schramm, T. Cowan, R. Sauerbrey, M. Bussmann, Picongpu: A fully relativistic particle-in-cell code for a gpu cluster, Plasma Science, IEEE Transactions on 38 (2010) 2831-2839.

[41] M. Bussmann, F. Schmitt, U. Schramm, J. Schuchart, R. Widera, H. Burau, T. E. Cowan, A. Debus, A. Huebl, G. Juckeland, T. Kluge, W. E. Nagel, R. Pausch, Radiative signatures of the relativistic Kelvin-Helmholtz instability, in: Proceedings of the International Conference for High Performance Computing, Networking, Storage and Analysis on - SC '13, ACM Press, New York, New York, USA, 2013 , pp. 1-12. URL: http: //dl . acm.org/citation. cfm?doid=2503210.2504564. doi:10.1145/2503210.2504564.

[42] F. Rossi, P. Londrillo, A. Sgattoni, S. Sinigardi, G. Turchetti, Towards robust algorithms for current deposition and dynamic load-balancing in a GPU particle in cell code, in: AIP Conf. Proc., volume 1507, AIP Publishing, 2012, pp. 184-192. URL: http://scitation.aip.org/content/aip/proceeding/aipcp/10.1063/1.4773692. doi:10.1063/1.4773692.

[43] H. Edwards, C. Trott, Kokkos: Enabling performance portability across manycore architectures, Extreme Scaling Workshop (XSW), (2013).

[44] H. Cheng, L. Greengard, V. Rokhlin, A fast adaptive multipole algorithm in three dimensions, Journal of Computational Physics 155 (1999) $468-498$.

[45] R. D. Ferraro, P. C. Liewer, V. K. Decyk, Dynamic load balancing for a 2d concurrent plasma PIC code, Journal of Computational Physics 109 (1993) $329-341$.

[46] A. Pukhov, Three-dimensional electromagnetic relativistic particle-in-cell code vlpl (virtual laser plasma lab), Journal of Plasma Physics 61 (1999) 425-433.

[47] E. Saule, E. Ö. Baş, Ü. V. Çatalyürek, Load-balancing spatially located computations using rectangular partitions, Journal of Parallel and Distributed Computing 72 (2012) $1201-1214$.

[48] J.-L. Vay, P. Colella, A. Friedman, D. Grote, P. McCorquodale, D. Serafini, Implementations of mesh refinement schemes for Particle-In-Cell plasma simulations, Computer Physics Communications 164 (2004) 297-305.

[49] D. Hilbert, Ueber die stetige abbildung einer line auf ein flächenstück, Mathematische Annalen 38 (1891) $459-460$.

[50] G. Peano, Sur une courbe, qui remplit toute une aire plane, Mathematische Annalen 36 (1890) 157-160. 
[51] K. Germaschewski, A. Bhattacharjee, R. Grauer, D. Keyes, B. Smith, Using krylov-schwarz methods in an adaptive mesh refinement environment, in: T. Plewa, T. Linde, V. G. Weirs (Eds.), Adaptive Mesh Refinement - Theory and Applications, Springer, 2005 , pp. 115-124.

[52] B. van der Holst, R. Keppens, Z. Meliani, A multidimensional grid-adaptive relativistic magnetofluid code, Computer Physics Communications 179 (2008) $617-627$.

[53] A. Calder, B. Fryxell, T. Plewa, R. Rosner, L. Dursi, V. Weirs, T. Dupont, H. Robey, J. Kane, B. Remington, R. Drake, G. Dimonte, M. Zingale, F. Timmes, K. Olson, P. Ricker, P. Macneice, H. Tufo, On validating an astrophysical simulation code, Astrophysical Journal, Supplement Series 143 (2002) 201-229. Cited By (since 1996)112. 